\title{
A review on sequential injection methods for water analysis
}

\author{
Raquel B.R. Mesquita, António O.S.S. Rangel* \\ CBQF/Escola Superior de Biotecnologia, Universidade Católica Portuguesa, R. Dr. António Bernardino de Almeida, 4200-072 Porto, Portuga
}

* Corresponding author. Tel.: +351 225580064; fax: +351 225090351

E-mail address: aorangel@esb.ucp.pt (A.O.S.S. Rangel).

Keywords:

Sequential injection

Lab-on-valve

Bead injection

Water analysis

Review

A B S T R A C T

The development of fast, automatic and less expensive methods of analysis has always been the main aim of flow methodologies. The search for new procedures that still maintain the reliability and accuracy of the reference procedures is an ever growing challenge. New requirements are continually added to analytical methodologies, such as lower consumption of samples and reagents, miniaturisation and portability of the equipment, computer interfaces for full decision systems and so on. Therefore, the development of flow methodologies meeting the extra requirements of water analysis is a challenging work.

Sequential injection analysis (SIA) presents a set of characteristics that make it highly suitable for water analysis. With sequential injection analysis, most routine determinations in waters can be performed more quickly with much lower reagent consumption when compared to reference procedures. Additionally, SIA can be a valuable tool for analyte speciation and multiparametric analysis. This paper critically reviews the overall work in this area.

\section{Introduction}

Water is an essential resource that is being threatened by pollution; therefore, the monitoring of water quality has become an issue of vital importance. The European Union has created the Water Framework Directive (WFD) in order to improve, protect and 
prevent further deterioration of water quality across Europe [1]. Accurate and frequent monitoring of water quality enables tighter control of the governmental regulations which is an important step in the reduction of the water pollution. It is estimated that, in European surface waters, the impact of pollution caused by industrial discharges of toxic substances has decreased $70 \%$ over the past 30 years [2]. This reduction has resulted from the implementation of stricter governmental regulations as much as the development of cleaner technologies.

The term "water" used in WFD includes most types of water, i.e. ground, surface and coastal waters. Several "water quality elements" are covered such as:

- Physicochemical properties - temperature, density, colour, turbidity, $\mathrm{pH}$ value, redox potential, conductivity, surface tension, suspended solids, total/dissolved organic carbon;

- Hydromorphological status - erosion and bench river characteristics;

- Biological - distribution and composition of the species and biological effects;

- Chemical monitoring - with particular emphasis on the contaminants in the list of priority pollutants.

Changes in water composition can be an indicator of pollution or contamination, so frequent water analysis is imperative. Some parameters are under strict regulation because of their risk to human health while others are not subject to enforceable regulation as they only cause visual or aesthetic effects [3]. More recently, the public concern for water safety has led to an increase on the use of disinfectants, which in turn has become a problem itself, if the carcinogenic by-products generated by the disinfectants are considered. Nevertheless, water disinfection is essential to ensure public health. Disinfectants prevent water contamination by bacteria but if in excess are harmful to human health, both directly and through by-products. In the last Analytical Chemistry biennial review on water analysis [4], the importance of disinfectants and their by-products as new contaminants was highlighted. These biennial reviews are focused on emerging contaminants and trends of analytical developments concerning water analysis.

The monitoring of water quality relies on effective routine water analysis, so this became a hot, sensitive and trendy issue. The range of methods, processes and tools available can be classified in different ways. A useful classification, revisited by Greenwood et al. [2], is based on the relationship between sample and analytical processes with three main categories: (i) in situ, (ii) on-line and (iii) off-line. Generally, the methods with no sampling, the methods with in-situ determination, use sensing devices such as probes inserted directly in the water body. Regarding on-line methods, the determination is carried out close to the water being monitored with direct feeding into the analytical system. In those cases, both discrete and continuous flow measurements can be performed.

Some major challenges in water quality monitoring include: a wide range of analyte concentration; variable salinity (wide range of ionic strength); colour or turbidity (mainly in waste waters); speciation; the need to mineralisation prior to quantifying the total amount. Analytes can be present in a high amount and so dilution may be necessary to fit the linear range, or they can be present in trace amounts and thus a preconcentration step is required. The variability in ionic strength may cause interferences in the chemistry itself or then influence the analytical signal, namely in spectrophotometric measurements. Water colour or turbidity may lead to high blank values which may adversely affect the limit of detection. The growing interest of environmental chemists in analyte speciation also poses an additional challenge, as some traditional methods only permit to quantify the total amount, namely when a reaction is used to quantify a certain analyte form may induce equilibrium shifts that unable to quantify the exact amount in that form. The interest of environmental chemists in analyte speciation relies on the tight relationship between speciation, bioavailability and toxicity. For example, aluminium is only bioaccumulated by organisms in the form of $\mathrm{Al}^{3+}$. Organisms use nitrate as nitrogen source while the nitrite form is highly toxic. Furthermore, changes in the environment caused by pollution such as environmental acidification result in the release of the cationic form of several metals, their most bioavailable form. The impact to human health becomes a consequence of the environmental impact. With the sequential injection versatility, this issue of analyte speciation can be tackled.

On the other hand, there are situations in which the quantification of the total amount is required and so drastic digestion conditions must be created. All these issues increase the complexity of water analysis.

In addition, the analysis of water should also try to comply with the objectives of so-called Green Chemistry [5], in order to reduce and/or eliminate the use and generation of hazardous substances. In fact, an ironic situation is created when the analytical methodologies employed to monitor pollutants generate chemical wastes that are highly polluting themselves [5]. In some cases, the chemicals used in the analysis are even more toxic than the analyte itself, which makes the search of new alternatives even more pertinent.

In this scenario, flow systems may provide answer to the above mentioned challenges, as virtually every unit operation can be implemented in-line, and offer several advantages for routine analysis namely, high sampling rate, miniaturisation, low sample and reagent consumption. The variety and capability of flow techniques and flow equipment has increased significantly over the last three decades. Scale of the equipment has gone from bench size equipment, handling volumes measured in liters, to coin size equipment with volumes in the order of microliters. This review focuses on the sequential injection analysis (SIA) approaches that meet some of these challenges, choosing sequential injection analysis [6] among the different automatic flow techniques (flow injection [7], multicommuted flow injection [8], multisyringe flow injection [9], multipumping flow [10]). This choice was determined by the robustness of the equipment along with the compact size, the possibility of multiparametric determinations and the low reagent consumption. In addition, the easy coupling of external devices such as gas diffusion or dialysis units, resin packed columns and so on, enables the application to a wide variety of waters. These features result from the versatility of the sequential injection valve which enables a direct connection to the various reagents and/or to external devices.

\section{Sequential injection analysis}

\section{Fundamentals}

Sequential injection was proposed as an evolution to flow injection analysis, to overcome some of its perceived disadvantages, the requirement for a separate manifold for the determination of each parameter and the continuous consumption of reagents. Other flow techniques such as multicommutation, multisyringe and multipumping overcome the continuous consumption of reagents but maintain, in many circumstances, the requirement of physical reconfiguration for different methodologies. Sequential injection has the ability of performing different determinations without system reconfiguration (placing different reagents on the ports of the selection valve) and there can be a reagent saving associated to non-continuous consumption. In a SIA manifold (Fig. 1(I)), sample and reagent solutions are sequentially aspirated into a holding coil, being the aspirated volumes determined by the time and aspiration 


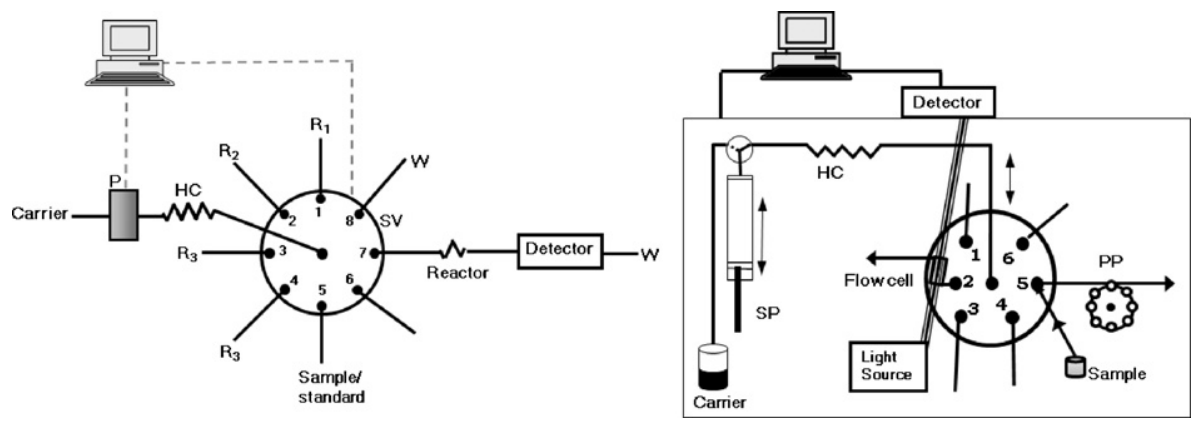

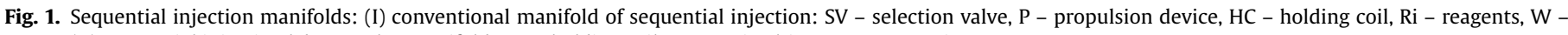
waste; (II) sequential injection lab-on-valve manifold: HC - holding coil, PP - peristaltic pump, SP - syringe pump.

rate; the mutual mixture is promoted by flow reversal, while sending the stacked zones towards the detection system. The computer control ensures the reproducibility of the process.

The basic components of sequential injection are schematically represented in Fig. 1(I). The key equipment is the multi-port selection valve as it enables the sequential selection of the various solutions and the subsequent redirection towards the detection system. Most of the characteristics attributed to sequential injection are due to the selection valve. In fact, the placement of different reagents on the ports permits different determinations with the same manifold and it is the structure of the selection valve itself that gives robustness to the sequential injection technique. The sequential aspiration of reagent and sample is made possible through the presence of the holding coil which prevents the contamination of the carrier. The propulsion system is usually a peristaltic pump or a piston pump and different detection systems can be used.

\section{Micro SIA (SI-LOV) and bead injection (BI)}

A step forward in miniaturisation and compaction of the sequential injection concept was recently achieved with micro sequential injection lab-on-valve (SI-LOV) equipment [11]. The SI-LOV concept was described as a universal micro-flow analyser based on the sequential injection concept. It resulted from the incorporation of the detection system in the selection valve which was made possible due to the use of fibre optics technology. With this significant down scale of sequential injection, there is an even lower consumption of reagents and samples. The micro sequential injection lab-on-valve concept is considered the third generation of flow analysis.

All the equipment, i.e. selection valve, propulsion device and detector, are assembled in the same box (Fig. 1(II)) resulting in the most compact of all the flow methodologies. As mentioned above, the volumes used are extremely small and the flow cell is positioned on the valve, which makes the analytical path rather short. The advantages in reagent and sample consumption minimisation are obvious and make SI-LOV a perfect tool for enzymatic and the so-called bead injection assays. The bead injection technique can be considered a "hyphenated" technique as it is commonly coupled with some other fluidic handling technique [12], where beads replace the reagent solution and the assay is carried on in the beads surface. This technique is especially suitable for immunoassays, coating the beads with antibodies.

\section{Evolution of the SIA application}

The features of simplicity and versatility allied with robustness have created an exponential growth of SIA applications. This growth in publications since SIA was first described in 1990, results not only from the relatively simple implementation but also the wide applicability of sequential injection techniques. From 1990, the cumulative number of papers increased to 286 in 2000, reaching 1204 papers last year (calculated using ISI Web of Knowledge Web of Science, keyword "sequential injection", 19/01/2009). The increase in SIA publications numbers is not, however comparable to flow injection and one of the reasons for that could be the requirement of computer control. This requirement implies some knowledge in computing for writing the programs as well as interfacing of the equipment used, which may be complex. Another reason could be the parallel development of other flow techniques (mentioned above) that have emerged from the original main concept of flow injection.

Along with all this increase in the diversity of flow manipulation, the possibilities in detection systems have also evolved extraordinarily. This evolution was not only in diversity but also in size, while fluid volumes have been reduced from mililiters to microliters and spectrophotometers have decreased in size about 80 fold. Therefore, the combination of flow handling techniques and detection systems has led to a countless number of possibilities. This may be the reason why there has also been an increase in the range of applications. Nowadays, flow systems have been developed for nearly all types of samples ranging from pharmaceutical preparations to complex solid samples such as soil and food. Based on the previously ISI Web of Knowledge - Web of Science search (Fig. 2) of sequential injection publications, a cross linked search with possible samples or types of sample enabled some conclusions to be drawn regarding the application of the described sequential injection systems.

First the types of sample were broadly categorized on environmental, food, pharmaceuticals or biological. Then, more specific classifications were used: wine, milk, water, plant extracts, soils and slurry. It is quite obvious that some overlap occurred as for example wine and milk are included in "food", and water and soil are included in "environmental", but the idea was to be as thorough as possible. "Biological samples" is the term commonly

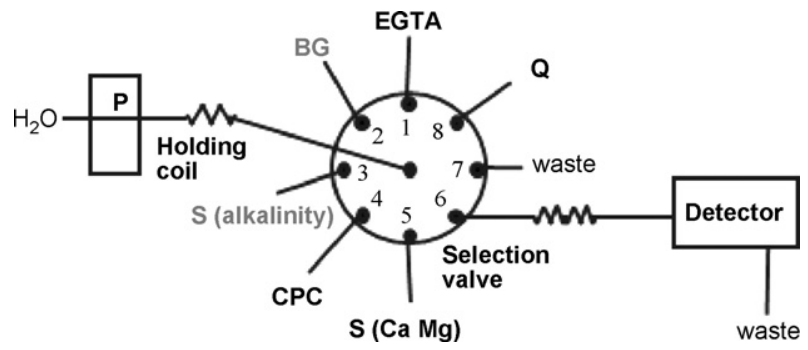

Fig. 2. Schematic representation of a SIA manifold for the multiparametric determination of calcium, magnesium and alkalinity in natural waters (adapted from [78]): $P$ - peristaltic pump, BG - bromocresol green reagent for alkalinity determination, Qhydroxiquinoline as masking agent for magnesium in calcium determination, EGTA - masking agent for calcium in magnesium determination, CPC - cresolphtalein complexone colour reagent for calcium and magnesium determination, $\mathrm{S}$ (alkalinity)/S (Ca, Mg) - sample for alkalinity or calcium and magnesium determination, respectively. 
used for samples as urine, blood and serum. In the end, almost half of all the described publications were not included in any category. Nevertheless, water samples are one of the three most common applications of the sequential injection technique along with pharmaceutical preparations and biological samples. It is a quite significant percentage which illustrates the concern for automation of water monitoring, especially when the other two main applications are health related, to pharmaceuticals and biological samples.

\section{Water analysis using SIA}

Introduction

As far as we know, no previous review was dedicated solely to sequential injection for water analysis. A review by Cerdà et al. [13] was dedicated to sequential injection applied to environmental samples, which include water, plants and soils. The papers [9,14-16] describe different flow methods for analysing waters and $[17,18]$ are dedicated to the application of flow injection to the same matrix.

In some reviews [19-21], the focus was on the evolution of flow methodologies, in which sequential injection was mentioned as a versatile and robust flow technique to couple with devices for inline treatments. Other reviews have focused on new developments in detection systems coupled with flow techniques [22-25], namely sequential injection. Some reviews of specific detection methods such as vibrational spectroscopy [22], FTIR [23], electrothermal atomic absorption spectroscopy [24] and electronic tongues [25] emphasised the versatility of flow techniques, including sequential injection.

It is also worth mentioning reviews on the determination of specific parameters/analytes (nutrients in aquatic systems [26], phosphorus determination [27]), or a specific type of sample (sea water analysis [28]).

The applications of the sequential injection concept to water analysis are presented in Table 1 . Molecular absorption spectrometry is by far the most commonly used detection method. This may be due to characteristics such as the robustness of the equipment, the possibility of miniaturisation, the versatility of application and the overall cost. Furthermore, it is a quite simple technique, not requiring any specific training. Other spectrophotometric detection systems such as flame emission atomic spectrometry (FEAS), flame atomic absorption spectrometry (FAAS), electrothermal atomic absorption spectrometry (ETAAS) and inductively coupled plasma mass spectrometry (ICP-MS) are generally more sensitive and selective, allowing trace elements analysis, but present a high maintenance costs and are not easily miniaturised. In addition, a more specific training is normally required. In the case of fluorescence and luminescence, the miniaturisation and cost are no longer a problem but the applicability is highly restricted. As for detection systems based in electroanalytical techniques such as potentiometry, amperometry and voltammetry, the portability and the in-situ determination are the main advantages. The problem with these techniques might be robustness and in some cases the limits of quantification.

One of the most common drawbacks attributed to sequential injection methods is the lower determination rate, especially when compared to flow injection. Nevertheless, that depends entirely on the method itself as it can be observed in Table 1 .

There is an extremely wide range of determination rates; from 3 determinations per hour in a methodology including separation and preconcentration [118] to 189 determinations per hour in a multiparametric determination [83]. The easy implementation of multiparametric determinations and diverse in-line sample treatments is a key attribute of sequential injection systems.
Water samples

More than $45 \%$ of the methods listed in Table 1 were applied to more than one type of water and $12 \%$ were applied to more than two types. This emphasises the versatility of sequential injection which can be applied to different types of water samples, by minor modifications in the flow programming, without any physical change of the manifold. Actually, some observations can be pointed out: (i) the significant percentage (over $6 \%$ ) of papers that did not specify the sample used; (ii) the percentage of papers that classified the sample as "natural water", close to $11 \%$, which include several types of water (sea, rain, river, lake, ground, mineral and tap water); and (iii) the percentage of papers that classified the sample as "surface water", close to $5 \%$, which also includes several types of water (sea, mineral river and lake). Tap water and waste water were the most analysed types with about 15\% each of the listed papers. Rain [118] and mine [136] waters were the least analysed, with only one methodology applied to each one.

\section{Multiparametric determinations}

One of the main advantages of sequential injection analysis is its potential to perform multiparametric analyses. This means that by using the same manifold but placing different reagents on the selection valve ports, and changing the operational parameters, more than one analyte can be determined. From the listed papers, over $40 \%$ involved the determination of more than one analyte $[34-36,39,40,45,49-53,55,59-61,72,74,78,83-85$, $94,96,101-104,106,107,114,116,117,119,120,132,135,138,140,141,145$, $148,149,153,155]$, with close to $18 \%$ describing the determination of more than two analytes [34-36,40,45,49-51,53,74,83,78,102,101, $116,140]$. Some more detailed examples of bi-parametric determinations will be detailed in Section 3.4.2, as they usually involve quantifying two oxidation states. When more than two analytes are considered, it usually implies the coupling to multi-analyte detection systems like ICP, HPLC or an array of sensors. This multiparametric capacity minimises the disadvantage of a low sample throughput that is often attributed to SIA. An example of a multiparametric SIA system with a determination rate of $40 \mathrm{~h}^{-1}$ for two parameters (calcium and magnesium) and $65 \mathrm{~h}^{-1}$ for a third one (alkalinity) is illustrated in Fig. 2.

\section{In-line treatments}

Another mentioned advantage of sequential injection is the ease coupling of separation devices to the valve, without overall manifold reconfiguration. This feature is due to the use of a selection valve, enabling the direct connection to the separation device. In fact, about $45 \%$ of all the listed papers described an in-line treatment of the sample. Within these papers, different types of in-line treatment are considered, some aiming for the separation and/or preconcentration of the analyte $(\approx 70 \%)$ and others aiming for the change of the oxidation state of the analyte $(\approx 30 \%)$.

\section{Separation and/or preconcentration}

Most of the works that include preconcentration and/or separation of the analyte refer to coupling SI methodology with ICP $[50,51,58,102]$ and AAS $[38,39,41,47,48,56,72,73,90,99,100$, $113,114,126,129,143]$. The combination of SIA with ICP or AAS associates the advantages of SIA such as versatility, robustness and automation (useful for handling reagent and sample preparation) with selectivity and sensitivity of those detection methods. The problems that normally arise from using a complex matrix were avoided by appropriate sample preparation, namely the inclusion of a preconcentration step; additionally, limits of detection of $1 \mathrm{pg} \mathrm{L}^{-1}$ were obtained [50]. 
Table 1

Sequential injection systems for water analysis.

\begin{tabular}{|c|c|c|c|c|c|c|c|c|c|}
\hline Year & Analyte & Sample & Detection system & Determination conditions & Dynamic range & LOD & RSD & $\begin{array}{l}\text { Determination } \\
\text { rate }\end{array}$ & Ref. \\
\hline 2008 & Methyl parathion & Surface water & Voltammetry & $\begin{array}{l}\text { SI for in-line sample } \\
\text { conditioning and standard } \\
\text { addition }\end{array}$ & $0.010-0.50 \mathrm{mg} \mathrm{L}^{-1}$ & $0.002 \mathrm{mg} \mathrm{L}^{-1}$ & $<4.5 \%$ & $25-61 \mathrm{~h}^{-1}$ & {$[29$} \\
\hline 2008 & $\begin{array}{l}\text { Linear alkylbenzene } \\
\text { sulfonates }\end{array}$ & Natural water & $\begin{array}{l}\text { Spectrophotometry/ } \\
\text { chromatography }\end{array}$ & $\begin{array}{l}\text { Lab-on-valve approach used } \\
\text { for application of two } \\
\text { detection methods }\end{array}$ & $0.07-10 \mu \mathrm{g} \mathrm{L}^{-1}$ & $\begin{array}{l}21 \mathrm{ng} \mathrm{L}^{-1} \\
15 \mu \mathrm{LL}^{-1}\end{array}$ & $<10.2 \%$ & $36-29 \mathrm{~h}^{-1}$ & [30] \\
\hline 2008 & Nitrite & Seawater & Spectrophotometry & $\begin{array}{l}\text { Griess reaction with solid } \\
\text { phase enrichment of the } \\
\text { coloured product prior to } \\
\text { detection }\end{array}$ & $\begin{array}{l}0.71-42.9 \mathrm{nM} \text { and } \\
35.7-429 \mathrm{nM}\end{array}$ & $0.1 \mathrm{nM}$ & $1.44 \%$ & $4 h^{-1}$ & [31] \\
\hline 2008 & Fluoride & Natural water & Potentiometry & $\begin{array}{l}\text { Comparison between a SI } \\
\text { system and a flow injection } \\
\text { system for the application of } \\
\text { a tubular electrode }\end{array}$ & $0.5-6.0 \mathrm{mg} \mathrm{L}^{-1}$ & $0.1 \mathrm{mg} \mathrm{L}^{-1}$ & $4 \%$ & $30 \mathrm{~h}^{-1}$ & [32] \\
\hline 2008 & Phosphorus & Sea water & Spectrophotometry & $\begin{array}{l}\text { Solid phase extraction with a } \\
\text { hydrophilic-lipophilic } \\
\text { balance used to enrich } \\
\text { phosphomolybdenium blue }\end{array}$ & $3.4-1132 \mathrm{nmol} \mathrm{L}^{-1}$ & $1.4 \mathrm{nmol} \mathrm{L}^{-1}$ & $2.5 \%$ & $6-10 h^{-1}$ & [33] \\
\hline 2008 & $\mathrm{Na}, \mathrm{K}$ and chloride & Mineral water & Potentiometry & $\begin{array}{l}\text { SI system with integrated } \\
\text { multisensor chip as base for } \\
\text { "electronic tongue" }\end{array}$ & $0.05 \mathrm{mM}-0.01 \mathrm{M}$ & - & $<8 \%$ & - & [34] \\
\hline 2008 & $\mathrm{Mg}, \mathrm{Ca}, \mathrm{Na}$ and $\mathrm{K}$ & Synthetic water & Potentiometry & $\begin{array}{l}\text { SI for automate the training } \\
\text { of the "electronic tongue" } \\
\text { employing the pulse } \\
\text { transient response }\end{array}$ & - & - & - & - & [35] \\
\hline 2008 & $\mathrm{Na}, \mathrm{K}$ and chloride & Mineral water & Potentiometry & $\begin{array}{l}\text { SI system based on a } \\
\text { multisensory ISFET array } \\
\text { monolithically integrated in } \\
\text { one chip }\end{array}$ & - & - & - & - & [36] \\
\hline 2008 & Al & Natural and waste water & Spectrophotometry & $\begin{array}{l}\text { A direct and kinetic } \\
\text { determination based in the } \\
\text { reaction with chrome } \\
\text { azurol S }\end{array}$ & $\begin{array}{l}0.040-0.500 \mathrm{mg} \mathrm{L}^{-1} \\
0.05-0.300 \mathrm{mg} \mathrm{L}^{-1}\end{array}$ & $0.002 \mathrm{mg} \mathrm{L}^{-1}, 0.012 \mathrm{mg} \mathrm{L}^{-1}$ & $\begin{array}{l}<5 \%, \\
<9 \%\end{array}$ & $\begin{array}{l}57 \mathrm{~h}^{-1} \\
31 \mathrm{~h}^{-1}\end{array}$ & [37] \\
\hline 2008 & $\mathrm{Cr}(\mathrm{III})$ and $\mathrm{Cr}(\mathrm{VI})$ & $\begin{array}{l}\text { Certified river water and } \\
\text { cave water }\end{array}$ & $\begin{array}{l}\text { Electrothermal atomic } \\
\text { absorption spectrometry }\end{array}$ & $\begin{array}{l}\text { Chromium speciation by } \\
\text { bio-sorption of } \mathrm{Cr}(\mathrm{VI}) \text { on } \\
\text { egg-shell membrane, } \mathrm{Cr}(\mathrm{III}) \\
\text { obtained by subtraction } \\
\text { (after conversion to } \mathrm{Cr}(\mathrm{VI})\end{array}$ & $0.05-1.25 \mu \mathrm{gL}^{-1} \mathrm{Cr}(\mathrm{VI})$ & $0.01 \mu \mathrm{g} \mathrm{L}^{-1} \mathrm{Cr}(\mathrm{VI})$ & $3.2 \%$ & $15 \mathrm{~h}^{-1}$ & [38] \\
\hline 2008 & $\mathrm{Cr}(\mathrm{VI})$ and $\mathrm{Cr}(\mathrm{III})$ & River and tap water & $\begin{array}{l}\text { Electrothermal atomic } \\
\text { absorption spectrometry }\end{array}$ & $\begin{array}{l}\text { Preconcentration and } \\
\text { speciation of chromium } \\
\text { using two mini-columns }\end{array}$ & $\begin{array}{l}0.1-0.25 \mu \mathrm{gL}^{-1} \mathrm{Cr}(\mathrm{III}) \\
0.12-2.0 \mu \mathrm{L}^{-1} \mathrm{Cr}(\mathrm{VI})\end{array}$ & $\begin{array}{l}0.02 \mathrm{Cr}(\mathrm{III}), 0.03 \mathrm{Cr}(\mathrm{VI}) \\
\left(\mu \mathrm{g} \mathrm{L}^{-1}\right)\end{array}$ & $\begin{array}{l}1.9 \% \mathrm{Cr}(\mathrm{III}), \\
2.5 \% \mathrm{Cr}(\mathrm{VI})\end{array}$ & $10 \mathrm{~h}^{-1}$ & [39] \\
\hline 2008 & $\mathrm{~Pb}, \mathrm{Cd}$ and $\mathrm{Zn}$ & Certified river water & Voltammetry & $\begin{array}{l}\text { Anodic stripping } \\
\text { voltammetry with a bismuth } \\
\text { film screen-printed carbon } \\
\text { electrode }\end{array}$ & $\begin{array}{l}0-70 \mu \mathrm{g} \mathrm{L}^{-1} \mathrm{~Pb} \text { e Cd, } \\
0.075-0.200 \mu \mathrm{g} \mathrm{L}^{-1} \mathrm{Zn}\end{array}$ & $\begin{array}{l}0.89 \mu \mathrm{g} \mathrm{Pb} \mathrm{L}^{-1}, \\
0.69 \mu \mathrm{gCd} \mathrm{L}^{-1}\end{array}$ & $<8.8 \%$ & - & [40] \\
\hline 2008 & $\mathrm{~Pb}(\mathrm{II})$ & Natural water & $\begin{array}{l}\text { Flame atomic absorption } \\
\text { spectrometry }\end{array}$ & $\begin{array}{l}\text { Liquid-liquid } \\
\text { micro-extraction for } \\
\text { preconcentration and/or } \\
\text { separation }\end{array}$ & $3.0-250.0 \mu \mathrm{g} \mathrm{L}^{-1}$ & $1.4 \mu \mathrm{g} \mathrm{L}^{-1}$ & $2.9 \%$ & $25 \mathrm{~h}^{-1}$ & [41] \\
\hline 2007 & $\begin{array}{l}\text { Alkylphenol } \\
\text { polyethoxylates }\end{array}$ & River water & Chemiluminescence & $\begin{array}{l}\text { Immunoassay with } \\
\text { microbeads }\end{array}$ & $0-1000 \mu \mathrm{g} \mathrm{L}^{-1}$ & $10 \mu \mathrm{g} \mathrm{L}^{-1}$ & - & $4 h^{-1}$ & [42] \\
\hline 2007 & Picloram & River and tap water & Squarewave voltammetry & Mercury drop electrode & $0.1-2.5 \mathrm{mg} \mathrm{L}^{-1}$ & $36 \mu g \mathrm{~L}^{-1}$ & $10.0 \%$ & $37 \mathrm{~h}^{-1}$ & [43] \\
\hline 2007 & Chlorine & Surface and tap water & Spectrophotometry & $\begin{array}{l}\text { Direct determination with as } \\
\text { a new application of }\end{array}$ & $0.09-1.3 \mathrm{mg} \mathrm{L}^{-1}$ & $80 \mu g \mathrm{~L}^{-1}$ & - & $60 \mathrm{~h}^{-1}$ & [44] \\
\hline
\end{tabular}




\begin{tabular}{|c|c|c|c|c|c|c|c|c|c|}
\hline Year & Analyte & Sample & Detection system & Determination conditions & Dynamic range & LOD & RSD & $\begin{array}{l}\text { Determination } \\
\text { rate }\end{array}$ & Ref. \\
\hline 2007 & $\mathrm{Mg}, \mathrm{Ca}, \mathrm{Ba}$ & Mineral water & Potentiometry & $\begin{array}{l}\text { Electronic tongue, } \\
\text { PVC-membrane } \\
\text { potentiometric sensor array } \\
\text { and multivariate calibration }\end{array}$ & $0-120 \mathrm{mg} \mathrm{Ca} \mathrm{L}^{-1}$ & - & - & - & [45] \\
\hline 2007 & $\mathrm{Hg}$ & $\begin{array}{l}\text { Certified river water and } \\
\text { sea water }\end{array}$ & $\begin{array}{l}\text { Atomic fluorescence } \\
\text { spectrometry }\end{array}$ & $\begin{array}{l}\text { Lab-on-valve approach with } \\
\text { a micro-scale vapor } \\
\text { generation chamber }\end{array}$ & $0.06-10 \mu g \mathrm{~L}^{-1}$ & $0.02 \mu \mathrm{gL}^{-1}$ & $4.4 \%$ & $90 \mathrm{~h}^{-1}$ & [46] \\
\hline 2007 & $\mathrm{Cd}$ & $\begin{array}{l}\text { Lake water and certified } \\
\text { river water }\end{array}$ & $\begin{array}{l}\text { Electrothermal atomic } \\
\text { absorption spectrometry }\end{array}$ & $\begin{array}{l}\text { Use of cell-sorption for } \\
\text { separation/preconcentration }\end{array}$ & $0.005-0.2 \mu \mathrm{g} \mathrm{L}^{-1}$ & $1.0 \mathrm{ng} \mathrm{L}^{-1}$ & $2.3 \%$ & $20 \mathrm{~h}^{-1}$ & [47] \\
\hline 2007 & $\mathrm{Cu}$ & $\begin{array}{l}\text { Coastal seawater and } \\
\text { certified seawater }\end{array}$ & $\begin{array}{l}\text { Electrothermal atomic } \\
\text { absorption spectrometry }\end{array}$ & $\begin{array}{l}\text { Sequential injection used for } \\
\text { sample pre-treatment and } \\
\text { concentration }\end{array}$ & $0.05-1.00 \mu \mathrm{g} \mathrm{L}^{-1}$ & $0.015 \mu g \mathrm{~L}^{-1}$ & $1.8 \%$ & $26 \mathrm{~h}^{-1}$ & [48] \\
\hline 2007 & $\mathrm{Co}, \mathrm{Ni}, \mathrm{Cu}, \mathrm{Fe}$ & Drinking waters & HPLC/spectrophotometry & $\begin{array}{l}\text { SI for automated handling of } \\
\text { sample/reagents, on-line } \\
\text { pre-column derivatization } \\
\text { and propulsion to HPLC }\end{array}$ & $\begin{array}{l}\text { From } 5-75 \mu g L^{-1} \text { to } \\
7-1000 \mu \mathrm{gL}^{-1}\end{array}$ & $\begin{array}{l}\text { From } 1 \mu \mathrm{gL}^{-1} \text { to } \\
2 \mu \mathrm{gL}^{-1}\end{array}$ & $6.0 \%$ & $4 h^{-1}$ & [49] \\
\hline 2007 & $\begin{array}{l}\mathrm{Be}, \mathrm{Cd}, \mathrm{Cr}, \mathrm{Cu} \mathrm{Pb} \text { and } \\
\text { other metals }\end{array}$ & $\begin{array}{l}\text { Certified river water and } \\
\text { tap water }\end{array}$ & ICP-MS or ICP-AES & $\begin{array}{l}\text { SI for sample preparation } \\
\text { and pre-treatment }\end{array}$ & $\begin{array}{l}\text { From } 0.001-10 \mathrm{ng} \mathrm{L}^{-1} \text { to } \\
0.1-10 \mathrm{ng} \mathrm{L}^{-1}\end{array}$ & $\begin{array}{l}\text { From } 0.001 \mathrm{ng} \mathrm{L}^{-1} \text { to } \\
018 \mathrm{ng} \mathrm{L}^{-1}\end{array}$ & $9.6 \%$ & $11 \mathrm{~h}^{-1}$ & [50] \\
\hline 2007 & $\begin{array}{l}\mathrm{Ag}, \mathrm{Be}, \mathrm{Cd}, \mathrm{Co}, \mathrm{Cu}, \mathrm{Ni}, \\
\mathrm{Pb}, \mathrm{U}, \mathrm{V}\end{array}$ & River water & ICP-MS & $\begin{array}{l}\text { Preconcentration of trace } \\
\text { elements }\end{array}$ & $\begin{array}{l}\text { From } 0.005-5 \mu \mathrm{gL}^{-1} \text { to } \\
1-5 \mu \mathrm{gL}^{-1}\end{array}$ & $\begin{array}{l}\text { From } 0.001 \mu \mathrm{gL}^{-1} \text { to } \\
0.93 \mu \mathrm{g} \mathrm{L}^{-1}\end{array}$ & $\begin{array}{l}\text { From } 0.2 \% \text { to } \\
1.2 \%\end{array}$ & - & [51] \\
\hline 2006 & Ammonia phosphate & River and marine water & Fluorimetry & $\begin{array}{l}\text { Free reactive phosphate } \\
\text { detected by suppression of } \\
\text { rhodamine fluorescence by } \\
\text { phosphomolybdate and } \\
\text { ammonia with phthaldehyde }\end{array}$ & $\begin{array}{l}0.02-20 \mu \mathrm{M} \\
0.04-4 \mu \mathrm{M}\end{array}$ & $\begin{array}{l}0.02 \mu \mathrm{M} \\
0.04 \mu \mathrm{M}\end{array}$ & - & $120 \mathrm{~h}^{-1}$ & [52] \\
\hline 2006 & $\begin{array}{l}\text { Chloride, nitrate, } \\
\text { hydrogencarbonate }\end{array}$ & $\begin{array}{l}\text { Well, spring and tap } \\
\text { water }\end{array}$ & Potentiometry & $\begin{array}{l}\text { Array of potentiometric } \\
\text { sensors and artificial neural } \\
\text { network, "electronic tongue" }\end{array}$ & - & - & $<7 \%$ & - & [53] \\
\hline 2006 & $\begin{array}{l}\text { Pharmaceutical } \\
\text { compounds }\end{array}$ & $\begin{array}{l}\text { Surface water and raw } \\
\text { and treated waste waters }\end{array}$ & HPLC/spectrophotometry & $\begin{array}{l}\text { Lab-on-valve as a front end } \\
\text { to HPLC with on-line solid } \\
\text { phase extraction }\end{array}$ & - & $0.05 \mu \mathrm{g} \mathrm{L}^{-1}$ & - & - & [54] \\
\hline 2006 & $\mathrm{As}(\mathrm{III})$ and $\mathrm{As}(\mathrm{V})$ & $\begin{array}{l}\text { Tap water and } \\
\text { groundwater }\end{array}$ & Chemiluminescence & $\begin{array}{l}\text { SI for fluidic handling for } \\
\text { measure waterborne arsenic }\end{array}$ & $0-50 \mu g \mathrm{~L}^{-1}$ & $0.05 \mu \mathrm{g} \mathrm{L}^{-1}$ & - & $15 \mathrm{~h}^{-1}$ & [55] \\
\hline 2006 & $\mathrm{Ni}$ & Tap and seawater & $\begin{array}{l}\text { Electrothermal atomic } \\
\text { absorption spectrometry }\end{array}$ & $\begin{array}{l}\text { Bead injection for } \\
\text { separation/preconcentration }\end{array}$ & $0.2-2 \mu \mathrm{g} \mathrm{L}^{-1}$ & $0.05 \mu \mathrm{gL}^{-1}$ & $4.8 \%$ & $10 \mathrm{~h}^{-1}$ & [56] \\
\hline 2006 & Se & River, lake and tap water & AAS-Hybrid & $\begin{array}{l}\text { SI for handling } \\
\text { sample/reagent }\end{array}$ & $0.10-8.0 \mu \mathrm{g} \mathrm{L}^{-1}$ & $0.03 \mu \mathrm{g} \mathrm{L}^{-1}$ & $2.8 \%$ & $24 \mathrm{~h}^{-1}$ & [57] \\
\hline 2006 & $\mathrm{~Pb}$ & $\begin{array}{l}\text { Reference river water \& river } \\
\text { samples }\end{array}$ & ICP-MS & $\begin{array}{l}\text { Preconcentration resin } V s=5 \\
\text { Preconcentration resin } \\
V s=10\end{array}$ & $0.1-5 \mathrm{ng} \mathrm{mL}^{-1}$ & $\begin{array}{l}70 \mathrm{pg} \mathrm{mL}^{-1} \\
30 \mathrm{pg} \mathrm{mL}^{-1}\end{array}$ & $0.5 \%$ & $12 \mathrm{~h}^{-1}$ & [58] \\
\hline 2006 & $\begin{array}{l}\mathrm{Cu}(\mathrm{II}) \\
\mathrm{Fe}\end{array}$ & $\begin{array}{l}\text { Standard solution and } \\
\text { industrial waste water }\end{array}$ & Spectrophotometry & $\begin{array}{l}\text { LOV, use of reducing agent } \\
\text { for determination of iron(II) }\end{array}$ & $\begin{array}{l}0.1-2 \mathrm{mg} \mathrm{L}^{-1} \\
0.1-5 \mathrm{mg} \mathrm{L}^{-1}\end{array}$ & $\begin{array}{l}50 \mu g \mathrm{~L}^{-1} \\
25 \mu \mathrm{gL}^{-1}\end{array}$ & $\begin{array}{l}2.0 \% \\
1.8 \%\end{array}$ & $18 \mathrm{~h}^{-1}$ & [59] \\
\hline 2006 & $\begin{array}{l}\mathrm{Cr}(\mathrm{VI}) \\
\mathrm{Cr}(\mathrm{III})\end{array}$ & Simulated water samples & $\begin{array}{l}\text { Spectrophotometry-diode } \\
\text { array }\end{array}$ & $\begin{array}{l}\text { Chemometric } \\
\text { tools-multivariate curve } \\
\text { resolution alternative least } \\
\text { squares (MCR-ALS) }\end{array}$ & $\begin{array}{l}0.02-0.5 \mathrm{mg} \mathrm{L}^{-1} \\
-\end{array}$ & $\begin{array}{l}2.4 \mu \mathrm{gL}^{-1} \\
-\end{array}$ & $\begin{array}{l}3.7 \% \\
4.9 \%\end{array}$ & $54 \mathrm{~h}^{-1}$ & [60] \\
\hline 2006 & $\begin{array}{l}\mathrm{Cr}(\mathrm{VI}) \\
\operatorname{Cr}(\mathrm{III})\end{array}$ & Simulated water samples & $\begin{array}{l}\text { Renewable surface reflection } \\
\text { spectrophotometry }\end{array}$ & $\begin{array}{l}\text { Reaction with } \mathrm{Cr}(\mathrm{IV}) \text { in beads } \\
\text { and oxidation of } \mathrm{Cr}(\mathrm{III}) \text { to } \\
\mathrm{Cr}(\mathrm{IV})\end{array}$ & $\begin{array}{l}0.02-0.5 \mathrm{mg} \mathrm{L}^{-1} \\
-\end{array}$ & $\begin{array}{l}2.4 \mu \mathrm{gL}^{-1} \\
-\end{array}$ & $\begin{array}{l}1.3 \% \\
2.5 \%\end{array}$ & $53 \mathrm{~h}^{-1}$ & [61] \\
\hline 2005 & $\begin{array}{l}\text { 3,5,6-trichloro-2- } \\
\text { pyridinol }\end{array}$ & $\begin{array}{l}\text { Tap water and river } \\
\text { water }\end{array}$ & $\begin{array}{l}\text { Electrochemical } \\
\text { immunoassay }\end{array}$ & $\begin{array}{l}\text { Magnetic beads coated with } \\
\text { antibody in a permanent } \\
\text { magnet reaction zone }\end{array}$ & $0.01-2 \mu \mathrm{g} \mathrm{L}^{-1}$ & $6 \mathrm{ng} \mathrm{L}^{-1}$ & $<3.9 \%$ & - & [62] \\
\hline 2005 & Cationic surfactants & Well and tap water & Spectrophotometry & $\begin{array}{l}\text { Reaction of cationic } \\
\text { surfactants as zephiramine } \\
\text { among others with } \\
\text { bromophenol blue }\end{array}$ & $7-50 \mu g L^{-1}$ & $0.22 \mu \mathrm{g} \mathrm{L}^{-1}$ & $1.8 \%$ & $28 \mathrm{~h}^{-1}$ & [63] \\
\hline
\end{tabular}




\begin{tabular}{|c|c|c|c|}
\hline 2005 & Cationic surfactants & Surface and waste waters & Spectrophotometry \\
\hline 2005 & Anionic surfactant & Drainage water sample & Spectrophotometry \\
\hline 2005 & $\begin{array}{l}p \text {-Arsenilic acid } \\
(p-\text { ASA })\end{array}$ & $\begin{array}{l}\text { Surface water from } \\
\text { swine farm }\end{array}$ & Spectrophotometry \\
\hline 2005 & Nitrite & Waste water treatment plant & Spectrophotometry \\
\hline 2005 & Atrazine & Waste and fresh water & Square wave voltammetry \\
\hline 2005 & Chlorine & $\begin{array}{l}\text { Tap water, waste water and } \\
\text { bleaches }\end{array}$ & Spectrophotometry \\
\hline 2005 & Chloride & $\begin{array}{l}\text { Mineral drinking water } \\
\text { and surface water }\end{array}$ & Potentiometry \\
\hline 2005 & $\mathrm{Fe}(\mathrm{III})$ & $\begin{array}{l}\text { Not specified water } \\
\text { samples }\end{array}$ & Spectrophotometry \\
\hline 2005 & $\begin{array}{l}\mathrm{Cr}(\mathrm{VI}) \\
\mathrm{Cr}(\mathrm{III})\end{array}$ & River, lake and tap water & $\begin{array}{l}\text { Electrothermal atomic } \\
\text { absorption spectrometry }\end{array}$ \\
\hline 2005 & $\mathrm{Cr}(\mathrm{VI})$ & $\begin{array}{l}\text { Seawater, tap water and } \\
\text { certified natural water }\end{array}$ & $\begin{array}{l}\text { Electrothermal atomic } \\
\text { absorption spectrometry }\end{array}$ \\
\hline 2005 & $\mathrm{Cu}, \mathrm{Fe}, \mathrm{Mn}, \mathrm{Zn}$ & $\begin{array}{l}\text { Natural, waste and } \\
\text { spiked water samples }\end{array}$ & Spectrophotometry \\
\hline 2004 & Bromate & $\begin{array}{l}\text { Not specified water } \\
\text { samples }\end{array}$ & Spectrophotometry \\
\hline 2004 & Boron & $\begin{array}{l}\text { Natural waters and } \\
\text { pharmaceuticals }\end{array}$ & Fluorimetry \\
\hline 2004 & Orthophosphate & $\begin{array}{l}\text { Waste water treatment } \\
\text { plant }\end{array}$ & Spectrophotometry \\
\hline 2004 & $\mathrm{Ca}, \mathrm{Mg}$ & $\begin{array}{l}\text { Drinking, surface, tap water } \\
\text { and reference water }\end{array}$ & Spectrophotometry \\
\hline & Alkalinity & & \\
\hline 2004 & Al & $\begin{array}{l}\text { Drinking water during } \\
\text { and after } \\
\text { flocculation/coagulation }\end{array}$ & Fluorimetry \\
\hline 2004 & $\mathrm{Hg}$ & River and sea water & $\begin{array}{l}\text { Cold-vapour atomic } \\
\text { absorption spectrometry }\end{array}$ \\
\hline 2004 & $\mathrm{~Pb}$ & Natural and waste water & Spectrophotometry \\
\hline 2004 & $\mathrm{~Pb}$ & Drinking water samples & Spectrophotometry \\
\hline 2004 & $\begin{array}{l}\mathrm{Pb}, \mathrm{Zn}, \mathrm{Co}, \mathrm{Cd}, \mathrm{Cu}, \\
\mathrm{Fe}(\mathrm{III}), \mathrm{Hg}\end{array}$ & $\begin{array}{l}\text { Soils, tap waters, urine } \\
\text { and certified samples }\end{array}$ & $\begin{array}{l}\text { Spectrophotometry-diode } \\
\text { array }\end{array}$ \\
\hline
\end{tabular}

Different colour reagents for
each determination
Liquid-liquid extraction, lab
at valve approach
Sequential injection-long
path length absorbance
spectrometry (SI-LPS)
Adaptative system for 2
ranges, automatically
programmable
SI for conditioning and
standard addition
Gas diffusion unit for
separation of free chloride,
colorimetric detection
Lab-at-valve approach with
the electrodes attached to
the multiposition valve
Home-made SIA,
Fe-thiocyanate complex
Bead injection for
separation/preconcentration
Bead injection with
renewable reverse phase for
separation/preconcentration
Different colour reagents for
each determination

to

Reaction between bromate and PADAP with thiocyanate Calibration curve based in boric acid and related to

On-line electrochemical on of molybdenum

Same reagent for $\mathrm{Ca}$ and $\mathrm{Mg}$ (cresolftaleine) with masking agents, alkalinity with bromocresol green

Separation of Al from matrix with XAD-4 (chelating resin) reaction with

hydroxyquinoline
Inclusion of a new integrated

Inclusion of a new integrate
gas-liquid separator

gas-liquid separator
operating in parallel as a

reactor

Preconcentration of $\mathrm{Pb}$ in

Chelex and interferences

removal in AG1X8 (resins)

Catalytic effect on reaction

with reazurin

Thin-film SI extraction with multivariate calibration and
8.10-6 to $1.10^{-4} \mathrm{M}$,

$1.10^{-4}$ to $5.10^{-4} \mathrm{M}$

$1-10 \mathrm{mg} \mathrm{L}^{-1}$

$0.1-1.0 \mathrm{mg} \mathrm{L}^{-1}$

0.0-3.0 $\mathrm{mg} \mathrm{L}^{-1}$

$0.0-20.0 \mathrm{mg} \mathrm{L}^{-1}$

$1.2 \times 10^{-7} \mathrm{M}$ to

$2.3 \times 10^{-6} \mathrm{M}$

$0.6-4.8 \mathrm{mg} \mathrm{L}^{-1}$

$0.047-0.188 \mathrm{gL}^{-1}$

$0.10-120 \mathrm{mM}$

$1.0-7.0 \mathrm{mg} \mathrm{L}^{-1}$

$0.035-0.4 \mu \mathrm{g} \mathrm{L}^{-1}$

$0.02-0.25 \mu \mathrm{g} \mathrm{L}^{-1}$

$0.12-1.5 \mu \mathrm{g} \mathrm{L}^{-1}$

$0-5 \mathrm{mg} \mathrm{L}^{-1}$,

$0-10 \mathrm{mg} \mathrm{L}^{-1}$,
$0-4 \mathrm{mg}^{-1}, 0-5 \mathrm{mg} \mathrm{L}^{-1}$

$0.18-3.00 \mathrm{mg} \mathrm{L}^{-1}$

$8-350 \mathrm{mg} \mathrm{L}^{-1}$

$0.3-20 \mathrm{mg} \mathrm{L}^{-1} \mathrm{P}$

$0.5-5 \mathrm{mg} \mathrm{Ca} \mathrm{L}^{-1}$

$0.5-10 \mathrm{mg} \mathrm{Mg} \mathrm{L}^{-1}$

$10-100 \mathrm{mg} \mathrm{L}^{-1} \mathrm{HCO}_{3}{ }^{-}$

$0.2-500 \mathrm{mg} \mathrm{L}^{-1}$

$0.05-5 \mu \mathrm{g} \mathrm{L}^{-1}$

$0.05-0.30 \mathrm{mg} \mathrm{L}^{-1}$

$0.30-1.0 \mathrm{mg} \mathrm{L}^{-1}$

0-0.100 mg

1-20 Co, Cu, Pb, 1-10 Hg

1-20 Zn, 1-20 Cd

$\left(\mathrm{mg} \mathrm{L}^{-1}\right)$
$2.20 .10^{-6} \mathrm{M}, 2.96 .10^{-4} \mathrm{M}$

$4.57 \%, 4.93 \%$

$29 \mathrm{~h}^{-1}, 28 \mathrm{~h}^{-1}$

$5 \mathrm{~h}^{-1}$

$7.5 \mathrm{~h}^{-1}$

$0.0212 \mathrm{mg} \mathrm{L}^{-1}$

$2.8 \%$

$12 \mathrm{~h}^{-1}$

$0.048 \mathrm{mg} \mathrm{L}^{-1}$

$0.4 \mathrm{mg} \mathrm{L}^{-1}$

$2.1 \times 10^{-6} \mathrm{M}$

$0.5 \mathrm{mg} \mathrm{L}^{-1}$

$5 \mathrm{mg} \mathrm{L}^{-1}$

$37 \mathrm{~h}^{-1}$

$15 \mathrm{~h}^{-1}$

$30 \mathrm{~h}^{-1}$

$50 \mathrm{~h}^{-1}$

$0.34 \mathrm{mg} \mathrm{L}^{-1}$

$1.3 \%$

$-$

$0.02 \mu \mathrm{g} \mathrm{L}^{-1}$

$0.01 \mu \mathrm{gL}^{-1}$

$0.03 \mu \mathrm{g} \mathrm{L}^{-1}$

$8 \mathrm{~h}^{-1}$
$12 \mathrm{~h}^{-1}$

$15 \mathrm{~h}^{-1}$

$0.048 \mathrm{mg} \mathrm{L}^{-1}$,

$0.012 \mathrm{mg} \mathrm{L}^{-1}$

$0.24 \mathrm{mg} \mathrm{L}^{-1}$

$0.15 \mathrm{mg} \mathrm{L}^{-1}$

$0.003 \mathrm{mg} \mathrm{L}^{-1}$

$2.2 \%$
$2.4 \%$

$$
3.8 \%
$$

$2.4 \%, 2.8 \%$,

$2.1 \%, 2 \%$

-

$0.8 \%$

$45 \mathrm{~h}^{-1}$

$49 h^{-1}$

$0.100 \mathrm{mg} \mathrm{L}^{-1} \mathrm{P}$

$0.32 \mathrm{mg} \mathrm{Ca} \mathrm{L}^{-1}$,

$5.1 \mathrm{mg} \mathrm{L}^{-1} \mathrm{HCO}_{3}{ }^{-}$

$2.0 \% \mathrm{Ca}, 2.1 \%$

$40+40 \mathrm{~h}^{-1}$

$65 \mathrm{~h}^{-1}$

$0.2 \mathrm{mg} \mathrm{L}^{-1}\left(\mathrm{~mL}^{-1}\right.$

$0.4 \%$

sample)

$0.02 \mu \mathrm{g} \mathrm{L}^{-1}$

$2.6 \%$

$25 \mathrm{~h}^{-1}$

$25 \mu \mathrm{gL}^{-1}$

$165 \mu \mathrm{g} \mathrm{L}^{-1}$

$17 \mathrm{~h}^{-1}$

$24 \mathrm{~h}^{-1}$

$27 \mathrm{~h}^{-1}$

$\left(189 \mathrm{dth}^{-1}\right)$ 
Table 1 (Continued)

\begin{tabular}{|c|c|c|c|c|c|c|c|c|c|}
\hline Year & Analyte & Sample & Detection system & Determination conditions & Dynamic range & LOD & RSD & $\begin{array}{l}\text { Determination } \\
\text { rate }\end{array}$ & Ref. \\
\hline 2003 & $\begin{array}{l}\text { Carbonate and } \\
\text { hydrogencarbonate }\end{array}$ & $\begin{array}{l}\text { Not specified water } \\
\text { samples }\end{array}$ & Spectrophotometry & $\begin{array}{l}\text { Titration using } \\
\text { phenolphthalein and methyl } \\
\text { orange }\end{array}$ & $\begin{array}{l}0.8-10 \mathrm{mM} \mathrm{CO}_{3}^{2-} \text {, } \\
1-10 \mathrm{mM} \mathrm{HCO}_{3}{ }^{-}\end{array}$ & - & $\begin{array}{l}2 \% \mathrm{CO}_{3}^{2-}, \\
1.5 \% \mathrm{HCO}_{3}-\end{array}$ & $12 \mathrm{~h}^{-1}$ & $\overline{[84]}$ \\
\hline 2003 & $\begin{array}{l}\text { Bromine } \\
\text { Bromide }\end{array}$ & $\begin{array}{l}\text { Spiked water samples and } \\
\text { effluents streams }\end{array}$ & Spectrophotometry & $\begin{array}{l}\text { Determination of bromine } \\
\text { and total bromine, oxidation, } \\
\text { (bromide by the difference) }\end{array}$ & $\begin{array}{l}1-10 \mathrm{mg} \mathrm{L}^{-1} \mathrm{Br}_{2} \\
0.8-15 \mathrm{mg} \mathrm{L}^{-1} \text { total } \mathrm{Br}_{2}\end{array}$ & $\begin{array}{l}0.6 \mathrm{mg} \mathrm{L}^{-1} \mathrm{Br}_{2} \\
0.4 \mathrm{mg} \mathrm{L}^{-1} \text { total } \mathrm{Br}_{2}\end{array}$ & $\begin{array}{l}0.8 \% \mathrm{Br}_{2} \\
0.7 \% \text { total } \mathrm{Br}_{2}\end{array}$ & $30 \mathrm{~h}^{-1}$ & [85] \\
\hline 2003 & sulphate & $\begin{array}{l}\text { Natural and waste } \\
\text { waters }\end{array}$ & Spectrophotometry & $\begin{array}{l}\text { Use of an air bubble for } \\
\text { minimise dispersion and } \\
\text { improve mixing, turbid } \\
\text { metric determination }(\mathrm{BaCl})\end{array}$ & $10-100 \mathrm{mg} \mathrm{L}^{-1}$ & $10 \mathrm{mg} \mathrm{L}^{-1}$ & $5 \%$ & $20 \mathrm{~h}^{-1}$ & {$[86]$} \\
\hline 2003 & Sulphide & Simulated water samples & Spectrophotometry & $\begin{array}{l}\text { Based in formation of } \\
\text { methylene blue dye, } \\
\text { calibration with in-line } \\
\text { dilution of a single standard }\end{array}$ & $0.17-1.0 \mathrm{mg} \mathrm{L}^{-1}$ & $0.04 \mathrm{mg} \mathrm{L}^{-1}$ & $5.20 \%$ & $38 \mathrm{~h}^{-1}$ & [87] \\
\hline 2003 & Tween-80 & $\begin{array}{l}\text { Well water, tap water } \\
\text { and seawater all spiked }\end{array}$ & Fluorimetry & $\begin{array}{l}\text { Enhancement of fluorescein } \\
\text { fluorescence in the presence } \\
\text { of Tween- } 80\end{array}$ & $10-2100 \mu \mathrm{g} \mathrm{L}^{-1}$ & $1.7 \mu \mathrm{g} \mathrm{L}^{-1}$ & $2.70 \%$ & - & [88] \\
\hline 2003 & Al & Tap water spiked & Fluorimetry & $\begin{array}{l}\text { Enhancement of the } \\
\text { fluorescence of the complex } \\
\text { aluminium-morin with } \\
\text { Tween-20 }\end{array}$ & $50-1000 \mu \mathrm{gL}^{-1}$ & $3 \mu g \mathrm{~L}^{-1}$ & $2.90 \%$ & - & [89] \\
\hline 2003 & $\mathrm{Cd}$ & $\begin{array}{l}\text { Reference river and } \\
\text { natural water }\end{array}$ & $\begin{array}{l}\text { Electrothermal atomic } \\
\text { absorption spectrometry }\end{array}$ & $\begin{array}{l}\text { Coupling bead injection with } \\
\text { lab-on-valve for on-line } \\
\text { matrix removal and } \\
\text { preconcentration }\end{array}$ & $0.05-1 \mu \mathrm{gL}^{-1}$ & $15 \mathrm{ng} \mathrm{L}^{-1}$ & $3.10 \%$ & $12 \mathrm{~h}^{-1}$ & [90] \\
\hline 2003 & $\mathrm{Cr}(\mathrm{VI})$ & $\begin{array}{l}\text { River and lake water } \\
\text { samples }\end{array}$ & Spectrophotometry & $\begin{array}{l}\text { Comparative studies of } \\
\text { diffusion samplers using } \\
\text { diphenylcarbazide (DPC) }\end{array}$ & $\begin{array}{l}0-1.6 \mathrm{mg} \mathrm{L}^{-1} \text { (without } \\
\text { membrane) }\end{array}$ & $20 \mathrm{mg} \mathrm{L}^{-1}$ & $1.10 \%$ & - & [91] \\
\hline 2003 & $\mathrm{Cu}$ & Mineral and tap water & Spectrophotometry & $\begin{array}{l}\text { Reaction with cuprizone, } \\
\text { sandwich SIA compared to } \\
\text { FIA }\end{array}$ & $0.06-4 \mathrm{mg} \mathrm{L}^{-1}$ & $0.004 \mathrm{mg} \mathrm{L}^{-1}$ & $0.71 \%$ & $48 \mathrm{~h}^{-1}$ & [92] \\
\hline 2003 & $\mathrm{Hg}$ (total) & $\begin{array}{l}\text { River water samples } \\
\text { spiked }\end{array}$ & Spectrophotometry & $\begin{array}{l}\text { Bead injection with Chelex } \\
\text { resin and dithizone for } \\
\text { colorimetric reaction }\end{array}$ & $0-30 \mathrm{mg} \mathrm{L}^{-1}$ & $0.9 \mathrm{mg} \mathrm{L}^{-1}$ & $9 \%$ & $20 \mathrm{~h}^{-1}$ & [93] \\
\hline 2003 & $\begin{array}{l}\mathrm{Mn}(\mathrm{II}) \\
\mathrm{Mn}(\mathrm{VII})\end{array}$ & $\begin{array}{l}\text { River water samples and } \\
\text { effluents streams }\end{array}$ & Spectrophotometry & $\begin{array}{l}\text { Determination of } \mathrm{Mn}(\mathrm{II}) \text { and } \\
\text { total Mn }(\mathrm{Mn}(\mathrm{VII}) \text { by the } \\
\text { difference) }\end{array}$ & $\begin{array}{l}0.02-0.50 \mathrm{mg} \mathrm{L}^{-1} \mathrm{Mn}(\mathrm{II}) \\
0.025-0.55 \mathrm{mg} \mathrm{L}^{-1} \text { total } \\
\mathrm{Mn}\end{array}$ & $\begin{array}{l}0.005 \mathrm{mg} \mathrm{L}^{-1} \mathrm{Mn}(\mathrm{II}) \\
0.008 \mathrm{mg} \mathrm{L}^{-1} \text { total } \mathrm{Mn}\end{array}$ & $\begin{array}{l}0.27 \% \mathrm{Mn}(\mathrm{II}) \\
0.34 \% \text { total } \\
\mathrm{Mn}\end{array}$ & $30 \mathrm{~h}^{-1}$ & [94] \\
\hline 2002 & Chloride & $\begin{array}{l}\text { Ground, surface and } \\
\text { waste water }\end{array}$ & Spectrophotometry & $\begin{array}{l}\text { Turbidimetric determination } \\
\text { based in the reaction } \\
\text { between silver nitrate and } \\
\text { chloride }\end{array}$ & $2-400 \mathrm{mg} \mathrm{L}^{-1}$ & $2 \mathrm{mgL}^{-1}$ & $3.7 \%$ & $55 h^{-1}$ & [95] \\
\hline 2002 & Nitrite nitrate & Surface water & Spectrophotometry & $\begin{array}{l}\text { Direct determination of } \\
\text { nitrite, reduction of nitrate to } \\
\text { nitrite in a } \\
\text { copperised-cadmium } \\
\text { column }\end{array}$ & $\begin{array}{l}0.05-1.00 \mathrm{mg} \mathrm{N} \mathrm{L}^{-1} \\
0.50-50.0 \mathrm{mg} \mathrm{N} \mathrm{L}^{-1}\end{array}$ & $\begin{array}{l}0.015 \mathrm{mg} \mathrm{N} \mathrm{L}^{-1} \\
0.10 \mathrm{mg} \mathrm{N} \mathrm{L}^{-1}\end{array}$ & $\begin{array}{l}1.10 \% \\
1.32 \%\end{array}$ & $14 \mathrm{~h}^{-1}$ & [96] \\
\hline 2002 & Fluorophores & Tap and mineral water & $\begin{array}{l}\text { Variable angle scanning } \\
\text { fluorescence spectrometry }\end{array}$ & $\begin{array}{l}\text { Multicomponent mixtures } \\
\text { coupling the detection with } \\
\text { multivariate least squares } \\
\text { regression }\end{array}$ & $\begin{array}{l}\text { From } 0.05-5 \mu \mathrm{g} \mathrm{L}^{-1} \text { to } \\
13-720 \mu \mathrm{g} \mathrm{L}^{-1}\end{array}$ & $\begin{array}{l}\text { From } 0.02 \mu \mathrm{gL}^{-1} \text { to } \\
10 \mu \mathrm{L}^{-1}\end{array}$ & $<1.0 \%$ & $17 \mathrm{~h}^{-1}$ & [97] \\
\hline 2002 & Al & Drinking and tap water & Spectrofluorimetry & $\begin{array}{l}\text { Reaction between } \mathrm{Al} \text { and } \\
\text { hydroxyquinoline and } \\
\text { fluorimetric detection of the } \\
\text { complex }\end{array}$ & $2.2-300 \mu \mathrm{g} \mathrm{L}^{-1}$ & $\mathrm{LOQ}=2.8 \mu \mathrm{g} \mathrm{L}^{-1}$ & $1.5 \%$ & $20 \mathrm{~h}^{-1}$ & [98] \\
\hline
\end{tabular}




\begin{tabular}{|c|c|c|c|c|c|c|c|c|}
\hline 2002 & $\mathrm{Cd}$ & Natural waters & $\begin{array}{l}\text { Electrothermal atomic } \\
\text { absorption spectrometry }\end{array}$ & $\begin{array}{l}\text { SI for on-line solvent } \\
\text { extraction-back extraction }\end{array}$ & $0.05-0.8 \mu \mathrm{g} \mathrm{L}^{-1}$ & $2.7 \mathrm{ng} \mathrm{L}^{-1}$ & $1.8 \%$ & $13 \mathrm{~h}^{-1}$ \\
\hline 2002 & $\mathrm{Cd}$ & Natural waters & $\begin{array}{l}\text { Electrothermal atomic } \\
\text { absorption spectrometry }\end{array}$ & $\begin{array}{l}\text { SI for on-line matrix removal } \\
\text { and preconcentration }\end{array}$ & $0.02-0.2 \mu g \mathrm{~L}^{-1}$ & $1.2 \mathrm{ng} \mathrm{L}^{-1}$ & $1.5 \%$ & $16 \mathrm{~h}^{-1}$ \\
\hline 2002 & $\mathrm{Cd}, \mathrm{Cu}, \mathrm{Pb}$ and $\mathrm{Zn}$ & $\begin{array}{l}\text { Drinking and waste } \\
\text { waters }\end{array}$ & Voltammetry & $\begin{array}{l}\text { Comparison of between a } \\
\text { flow injection system and a } \\
\text { sequential injection system } \\
\text { (still in study) }\end{array}$ & $\begin{array}{l}\text { From } 10-70 \mu g \mathrm{~L}^{-1} \text { to } \\
470-700 \mu \mathrm{L}^{-1}\end{array}$ & $\begin{array}{l}\text { From } 6 \mu \mathrm{g} \mathrm{L}^{-1} \text { to } \\
4700 \mu \mathrm{gL}^{-1}\end{array}$ & $<9.8 \%$ & - \\
\hline 2002 & $\begin{array}{l}\mathrm{Al}, \mathrm{As}, \mathrm{Co}, \mathrm{Cu}, \mathrm{Mn} \text {, } \\
\mathrm{Mo}, \mathrm{Ni}, \mathrm{Pb} \text { and } \mathrm{V}\end{array}$ & Sea water & ICP-MS & $\begin{array}{l}\text { Comparison of different } \\
\text { resins for preconcentration }\end{array}$ & $0-100 \mu g \mathrm{~L}^{-1}$ & - & $<5 \%$ & - \\
\hline 2002 & $\begin{array}{l}\mathrm{Fe}(\mathrm{III}) \\
\mathrm{Fe}(\mathrm{II})\end{array}$ & Effluents streams & Spectrophotometry & $\begin{array}{l}\text { Determination of } \mathrm{Fe}(\mathrm{III}) \text { and } \\
\text { total Fe, oxidation, (Fe(II) by } \\
\text { the difference) }\end{array}$ & $\begin{array}{l}0.15-100 \mathrm{mg} \mathrm{L}^{-1} \\
0.30-80 \mathrm{mg} \mathrm{L}^{-1}\end{array}$ & $\begin{array}{l}0.10 \mathrm{mg} \mathrm{L}^{-1} \\
0.15 \mathrm{mg} \mathrm{L}^{-1}\end{array}$ & $\begin{array}{l}1.3 \% \mathrm{Fe}(\mathrm{III}) \\
0.8 \% \mathrm{Fe}(\mathrm{II})\end{array}$ & $30 \mathrm{~h}^{-1}$ \\
\hline 2002 & $\begin{array}{l}\mathrm{Cr}(\mathrm{III}) \\
\mathrm{Cr}(\mathrm{VI})\end{array}$ & $\begin{array}{l}\text { Water samples (not } \\
\text { specified) }\end{array}$ & Spectrophotometry & $\begin{array}{l}\text { Direct determination of } \\
\mathrm{Cr}(\mathrm{VI}) \text { and total } \mathrm{Cr} \text { (oxidation } \\
\text { of } \mathrm{Cr}(\mathrm{III}) \text { to } \mathrm{Cr}(\mathrm{VI})), \mathrm{Cr}(\mathrm{III}) \text { by } \\
\text { the difference }\end{array}$ & $\begin{array}{l}0.85-25 \mathrm{mg} \mathrm{L}^{-1} \\
0.16-20 \mathrm{mg} \mathrm{L}^{-1}\end{array}$ & $\begin{array}{l}0.042 \mathrm{mg} \mathrm{L}^{-1} \\
0.023 \mathrm{mg} \mathrm{L}^{-1}\end{array}$ & $0.70 \%$ & $30 \mathrm{~h}^{-1}$ \\
\hline 2002 & ${ }^{90} \mathrm{Sr}$ & $\begin{array}{l}\text { Mineral, ground and } \\
\text { marine water }\end{array}$ & $\begin{array}{l}\text { Low-background gas-flow } \\
\text { proportional counter }\end{array}$ & $\begin{array}{l}\text { Si for on-line wetting-film } \\
\text { extraction and sample } \\
\text { handling }\end{array}$ & $0.07-0.30 \mathrm{~Bq}$ & - & $3.0 \%$ & - \\
\hline 2001 & $\begin{array}{l}\text { fluorberidazole } \\
\text { thiabenzadole }\end{array}$ & Natural water & $\begin{array}{l}\text { Scanning fluorescence } \\
\text { spectrometry }\end{array}$ & $\begin{array}{l}\text { Simultaneous determination } \\
\text { of both pesticides }\end{array}$ & $\begin{array}{l}0.04-10 \mu \mathrm{g} \mathrm{L}^{-1} \\
0.08-20 \mu \mathrm{g} \mathrm{L}^{-1}\end{array}$ & $\begin{array}{l}1 \mu g \mathrm{~L}^{-1} \\
0.02 \mu \mathrm{g} \mathrm{L}^{-1}\end{array}$ & $\begin{array}{l}0.30 \% \\
0.50 \%\end{array}$ & - \\
\hline 2001 & $\begin{array}{l}\text { Nitrogen } \\
\text { Phosphate }\end{array}$ & Lake and tap water & Spectrophotometry & $\begin{array}{l}\text { Determination of nitrite, } \\
\text { nitrate (reduced } \\
\text { copperised-cadmium } \\
\text { column) and orthophosphate }\end{array}$ & $\begin{array}{l}30-4000 \mu \mathrm{g} \mathrm{L}^{-1} \\
1.0-30.0 \mu \mathrm{g} \mathrm{L}^{-1}\end{array}$ & $\begin{array}{l}3.91 \mu \mathrm{g} \mathrm{L}^{-1} \\
9.92 \mu \mathrm{g} \mathrm{L}^{-1}\end{array}$ & $<1.22 \%$ & $48 h^{-1}$ \\
\hline 2001 & Oxidised nitrogen & Natural waters & Spectrophotometry & $\begin{array}{l}\text { Determination of } \\
\text { nitrate + nitrite as } \mathrm{N} \text { after } \\
\text { reduction of nitrate } \\
\text { (cadmium) }\end{array}$ & $0.0-5 \mathrm{mg} \mathrm{L}^{-1}$ & $0.01 \mathrm{mg} \mathrm{L}^{-1}$ & $1.20 \%$ & $36 \mathrm{~h}^{-1}$ \\
\hline 2001 & Chloride & $\begin{array}{l}\text { Mineral and drinking } \\
\text { waters }\end{array}$ & Spectrophotometry & $\begin{array}{l}\text { Determination based on the } \\
\text { thiocyanate method, } \\
\text { formation of red iron(III) } \\
\text { thiocyanate complex }\end{array}$ & $0-50 \mathrm{mg} \mathrm{L}^{-1}$ & $3.01 \mathrm{mg} \mathrm{L}^{-1}$ & $2.5 \%$ & $37 h^{-1}$ \\
\hline 2001 & Iodide & $\begin{array}{l}\text { Drinking water samples } \\
\text { and pharmaceuticals }\end{array}$ & Spectrophotometry & $\begin{array}{l}\text { Based in the catalytic effect } \\
\text { of iodide on redox reaction } \\
\text { between } \mathrm{Ce}(\mathrm{IV}) \text { and } \mathrm{As}(\mathrm{III})\end{array}$ & $1-60 \mathrm{mg} \mathrm{L}^{-1}$ & $1.5 \mathrm{mg} \mathrm{L}^{-1}$ & - & $15 \mathrm{~h}^{-1}$ \\
\hline 2001 & benzo[A]pyrene & Tap and distilled water & $\begin{array}{l}\text { Variable angle fluorescence } \\
\text { spectrometry }\end{array}$ & $\begin{array}{l}\text { Extraction and } \\
\text { preconcentration prior to } \\
\text { detection }\end{array}$ & $7.5-280 \mathrm{ng} \mathrm{L}^{-1}$ & $2.5 \mathrm{ng} \mathrm{L}^{-1}$ & $1.10 \%$ & $4.5 \mathrm{~h}^{-1}$ \\
\hline 2001 & $\mathrm{Hg}$ & River water & $\begin{array}{l}\text { Cold-vapour atomic } \\
\text { absorption spectrometry }\end{array}$ & $\begin{array}{l}\text { Standard addition SI method } \\
\text { with on-line UV-digestion }\end{array}$ & $20-1000 \mathrm{ng} \mathrm{L}^{-1}$ & - & $5-30 \%$ & - \\
\hline 2001 & $\mathrm{Ni}$ & Waste water & $\begin{array}{l}\text { Electrothermal atomic } \\
\text { absorption spectrometry }\end{array}$ & $\begin{array}{l}\text { Coupling SI in-line } \\
\text { preconcentration with } \\
\text { renewable micro column } \\
\text { ion-exchange beads }\end{array}$ & $0.02-1.20 \mu \mathrm{g} \mathrm{L}^{-1}$ & $10.2 \mathrm{ng} \mathrm{L}^{-1}$ & $5.80 \%$ & $12 \mathrm{~h}^{-1}$ \\
\hline 2001 & $\begin{array}{l}\mathrm{Cr}(\mathrm{III}) \\
\mathrm{Cr}(\mathrm{VI})\end{array}$ & Effluent and natural water & $\begin{array}{l}\text { Flame atomic absorption } \\
\text { spectroscopy }\end{array}$ & $\begin{array}{l}\text { Speciation of chromium with } \\
\text { anionic and cationic resins }\end{array}$ & $\begin{array}{l}0.5-1.5 \mathrm{mg} \mathrm{L}^{-1} \\
-\end{array}$ & $\begin{array}{l}81 \mu g L^{-1} \\
42 \mu g L^{-1}\end{array}$ & $<10 \%$ & $12 \mathrm{~h}^{-1}$ \\
\hline 2000 & Boron & $\begin{array}{l}\text { Liquid fertilizers and } \\
\text { effluent water samples }\end{array}$ & Spectrophotometry & $\begin{array}{l}\text { In-situ preparation of } \\
\text { azomethine-H } \\
\text { (salicyaldehyde and H-acid } \\
\text { in boron's presence) }\end{array}$ & $0.61-100 \mathrm{mg} \mathrm{L}^{-1}$ & $0.61 \mathrm{mg} \mathrm{L}^{-1}$ & $1.40 \%$ & $30 h^{-1}$ \\
\hline 2000 & $\begin{array}{l}\text { Nitrite, nitrate, } \\
\text { sulphate, phenolic } \\
\text { compounds }\end{array}$ & Waste waters & Spectrophotometry & $\begin{array}{l}\text { Griess reaction for nitrite, } \\
\text { nitrate reduced (Cd column), } \\
\text { turbidimetry for sulphate, } \\
\text { phenolic compounds } \\
\text { through oxidative properties }\end{array}$ & $\begin{array}{l}\text { From } 0.05-15 \mathrm{mg} \mathrm{L}^{-1} \text { to } \\
5-200 \mathrm{mg} \mathrm{L}^{-1}\end{array}$ & $\begin{array}{l}\text { From } 0.03 \mathrm{mg} \mathrm{L}^{-1} \text { to } \\
19 \mathrm{mg} \mathrm{L}^{-1}\end{array}$ & $<2.2 \%$ & $12 \mathrm{~h}^{-1}$ \\
\hline
\end{tabular}

[99]

phenolic compounds

through oxidative properties 


\begin{tabular}{|c|c|c|c|c|c|c|c|c|c|}
\hline Year & Analyte & Sample & Detection system & Determination conditions & Dynamic range & LOD & RSD & $\begin{array}{l}\text { Determination } \\
\text { rate }\end{array}$ & Ref. \\
\hline 2000 & $\begin{array}{l}\text { Nitrite } \\
\text { Nitrate }\end{array}$ & Waste waters & Spectrophotometry & $\begin{array}{l}\text { Griess reaction for nitrite, } \\
\text { nitrate reduced to nitrite } \\
\text { (copperised cadmium } \\
\text { column) }\end{array}$ & $\begin{array}{l}0.05-25 \mathrm{mg} \mathrm{L}^{-1} \\
0.05-15 \mathrm{mg} \mathrm{L}^{-1}\end{array}$ & $\begin{array}{l}0.01 \mathrm{mg} \mathrm{L}^{-1} \\
0.01 \mathrm{mg} \mathrm{L}^{-1}\end{array}$ & $\begin{array}{l}2.0 \% \\
1.3 \%\end{array}$ & $24 \mathrm{~h}^{-1}$ & $\overline{[117]}$ \\
\hline 2000 & Nitrite & $\begin{array}{l}\text { Rain, tap water, ground, pond } \\
\text { and sea water }\end{array}$ & Spectrophotometry & $\begin{array}{l}\text { Isolation, preconcentration } \\
\text { and determination of nitrite } \\
\text { based on the Shinn reaction }\end{array}$ & $\begin{array}{l}13.4-160 \mathrm{mg} \mathrm{L}^{-1} \\
0.83-20 \mathrm{mg} \mathrm{L}^{-1}\end{array}$ & $\begin{array}{l}5.9 \mathrm{mg} \mathrm{L}^{-1} \\
0.32 \mathrm{mg} \mathrm{L}^{-1}\end{array}$ & $4.00 \%$ & $\begin{array}{l}15 \mathrm{~h}^{-1} \\
3 \mathrm{~h}^{-1}\end{array}$ & [118] \\
\hline 2000 & $\begin{array}{l}\text { Phosphate } \\
\text { Silicate }\end{array}$ & $\begin{array}{l}\text { River reservoir water } \\
\text { samples, sediments and } \\
\text { culture mediums }\end{array}$ & Spectrophotometry & $\begin{array}{l}\text { Reaction with molybdenum } \\
\text { blue avoid interferences of } \\
\text { each other with oxalic acid }\end{array}$ & $\begin{array}{l}0.2-7 \mathrm{mg} \mathrm{L}^{-1} \\
5-50 \mathrm{mg} \mathrm{L}^{-1}\end{array}$ & $\begin{array}{l}0.1 \mathrm{mg} \mathrm{L}^{-1} \\
1 \mathrm{mg} \mathrm{L}^{-1}\end{array}$ & - & $\begin{array}{l}75 h^{-1} \\
40 h^{-1}\end{array}$ & [119] \\
\hline 2000 & Phosphate and silicate & Waste waters & $\begin{array}{l}\text { Spectrophotometry-diode } \\
\text { array }\end{array}$ & $\begin{array}{l}\text { Determination based on } \\
\text { different reaction rates of ... } \\
\text { of phosphomolybdenium } \\
\text { blue }\end{array}$ & $\begin{array}{l}0.026-0.485 \text { P, } 0.125-2.848 \\
\left.\text { Si (mmol L }{ }^{-1}\right)\end{array}$ & 7.4 P, $37.38 \mathrm{Si}\left(\mathrm{mmol} \mathrm{L}^{-1}\right)$ & $\begin{array}{l}2.10 \% \\
1.10 \%\end{array}$ & $30 \mathrm{~h}^{-1}$ & {$[120]$} \\
\hline 2000 & Sulphuric acid & Effluents & Spectrophotometry & $\begin{array}{l}\text { SI titration with sodium } \\
\text { hydroxide }\end{array}$ & $0.006-0.178 \mathrm{M}$ & $0.002 \mathrm{M}$ & $<0.75 \%$ & $23 \mathrm{~h}^{-1}$ & [121] \\
\hline 2000 & Sulphate & Waste waters & $\begin{array}{l}\text { Ultraviolet- } \\
\text { spectrophotometry }\end{array}$ & $\begin{array}{l}\text { Formation of a cation } \\
\text { between iron and sulphate }\end{array}$ & $10-1000 \mathrm{mg} \mathrm{L}^{-1}$ & $5 \mathrm{mg} \mathrm{L}^{-1}$ & $2.40 \%$ & $72 \mathrm{~h}^{-1}$ & {$[122]$} \\
\hline 2000 & Thiocyanate & $\begin{array}{l}\text { Waste water samples } \\
\text { from effluent streams }\end{array}$ & Spectrophotometry & $\begin{array}{l}\text { Reaction between } \\
\text { thiocyanate and iron(III), } \\
\text { formation of the coloured } \\
\text { complex }\end{array}$ & $2.0-150 \mathrm{mg} \mathrm{L}^{-1}$ & $1.1 \mathrm{mg} \mathrm{L}^{-1}$ & $1.20 \%$ & $24 \mathrm{~h}^{-1}$ & [123] \\
\hline 2000 & $\mathrm{Hg}$ & River water & $\begin{array}{l}\text { Cold-vapour atomic } \\
\text { absorption spectrometry }\end{array}$ & $\begin{array}{l}\text { SI for standard addition } \\
\text { method, on-line } \\
\text { UV-digestion and sample } \\
\text { handling }\end{array}$ & $20-1000 \mathrm{ng} \mathrm{L}^{-1}$ & $10 \mathrm{ng} \mathrm{L}^{-1}$ & $<10 \%$ & $3 \mathrm{~h}^{-1}$ & [124] \\
\hline 2000 & $\mathrm{Hg}$ (total) & $\begin{array}{l}\text { Lake and tap water and } \\
\text { tuna samples }\end{array}$ & $\begin{array}{l}\text { Cold-vapour atomic } \\
\text { absorption spectrometry }\end{array}$ & $\begin{array}{l}\text { Improvement in comparing } \\
\text { with FI with same detection }\end{array}$ & $1.0-20 \mathrm{mg} \mathrm{L}^{-1}$ & $0.46 \mathrm{mg} \mathrm{L}^{-1}$ & $0.90 \%$ & $45 \mathrm{~h}^{-1}$ & [125] \\
\hline 2000 & $\mathrm{Cd}$ & $\begin{array}{l}\text { Drinking, mineral, river } \\
\text { and sea water }\end{array}$ & $\begin{array}{l}\text { Electrothermal atomic } \\
\text { absorption spectrometry }\end{array}$ & $\begin{array}{l}\text { SI for on-line solvent } \\
\text { extraction }\end{array}$ & $0-80 \operatorname{ng~L}^{-1}$ & $0.5 \mathrm{ng} \mathrm{L}^{-1}$ & $2.1 \%$ & $12 \mathrm{~h}^{-1}$ & [126] \\
\hline 2000 & $\mathrm{Fe}$ (total), as $\mathrm{Fe}(\mathrm{III})$ & $\begin{array}{l}\text { Pharmaceuticals, natural } \\
\text { waters and effluent } \\
\text { streams }\end{array}$ & Spectrophotometry & $\begin{array}{l}\text { Reduction of } \mathrm{Fe}(\mathrm{III}) \text { to } \mathrm{Fe}(\mathrm{II}) \\
\text { with cadmium and reaction } \\
\text { of } \mathrm{Fe}(\mathrm{II}) \text { with } \\
\text { 1,10-phenanthroline }\end{array}$ & $0.002-0.50 \mathrm{mg} \mathrm{L}^{-1}$ & $0.18 \mathrm{mg} \mathrm{L}^{-1}$ & $2.50 \%$ & $24 \mathrm{~h}^{-1}$ & [127] \\
\hline 2000 & $\mathrm{Mn}(\mathrm{II})$ & Tap water & Spectrophotometry & $\begin{array}{l}\text { Use of a solid-phase lead(IV) } \\
\text { dioxide reactor }\end{array}$ & $1-7 \mathrm{mg} \mathrm{L}^{-1}$ & $062 \mathrm{mg} \mathrm{L}^{-1}$ & $3.0 \%$ & $50 \mathrm{~h}^{-1}$ & [128] \\
\hline 1999 & $\mathrm{~Pb}$ & Natural waters & $\begin{array}{l}\text { Flame atomic absorption } \\
\text { spectroscopy }\end{array}$ & $\begin{array}{l}\mathrm{Pb} \text { preconcentration in } \\
\text { poly(vinylpyrrolidone) - PVP, } \\
\text { coupled SIA/FAAS }\end{array}$ & $11.8-400 \mathrm{mg} \mathrm{L}^{-1}$ & $4.9 \mathrm{mg} \mathrm{L}^{-1}$ & $3.0 \%$ & $16 \mathrm{~h}^{-1}$ & [129] \\
\hline 1999 & $\mathrm{Cu}$ & $\begin{array}{l}\text { Food samples (water } \\
\text { soluble) and water } \\
\text { samples }\end{array}$ & Spectrophotometry & $\begin{array}{l}\text { Based on the reaction } \\
\text { between Cu(II) with } \\
\text { diethyldithiocarbamate } \\
\text { (DDTC) }\end{array}$ & $0.5-5.0 \mathrm{mg} \mathrm{L}^{-1}$ & $0.2 \mathrm{mg} \mathrm{L}^{-1}$ & $4.5 \%$ & $7 h^{-1}$ & [130] \\
\hline 1998 & Nitrite & $\begin{array}{l}\text { Feed, dam and waste } \\
\text { water, effluent stream } \\
\text { and fertilizer }\end{array}$ & Spectrophotometry & $\begin{array}{l}\text { Nitrite is diatonised with } \\
N-(1- \\
\text { naphthyl)ethylenediammonium } \\
\text { dichloride resulting a } \\
\text { coloured azo dye }\end{array}$ & $0.05-5.0 \mathrm{mg} \mathrm{L}^{-1}$ & $0.053 \mathrm{mg} \mathrm{L}^{-1}$ & $2.63 \%$ & $49 \mathrm{~h}^{-1}$ & {$[131]$} \\
\hline 1998 & Nitrate and nitrite & $\begin{array}{l}\text { Tap, mineral and sea } \\
\text { water }\end{array}$ & $\begin{array}{l}\text { Spectrophotometry-diode } \\
\text { array }\end{array}$ & $\begin{array}{l}\text { Sandwich arrangement with } \\
\text { Griess reagent (nitrate } \\
\text { reduced to nitrite - } \\
\text { cadmium) }\end{array}$ & $\begin{array}{l}0.5-40 \mathrm{NO}_{2}^{-}, 2-100 \\
\mathrm{NO}_{3}^{-}\left(\mathrm{mmol} \mathrm{NL}^{-1}\right)\end{array}$ & $\begin{array}{l}0.1 \mathrm{NO}_{2}^{-}, 0.45 \mathrm{NO}_{3}^{-} \\
\left(\mathrm{mmol} \mathrm{N} \mathrm{L}^{-1}\right)\end{array}$ & $<2 \%$ & $10 \mathrm{~h}^{-1}$ & [132] \\
\hline 1998 & Warfarin & $\begin{array}{l}\text { Domestic water samples } \\
\text { spiked }\end{array}$ & Fluorimetry & $\begin{array}{l}\text { Enhancement of the } \\
\text { fluorescence resulting from } \\
\text { the complex with } \\
\text { b-cyclodextrin }\end{array}$ & $0.1-1 \mathrm{mg} \mathrm{L}^{-1}$ & $0.02 \mathrm{mg} \mathrm{L}^{-1}$ & $1.50 \%$ & $26 \mathrm{~h}^{-1}$ & [133] \\
\hline
\end{tabular}




\begin{tabular}{|c|c|c|c|c|c|c|c|c|}
\hline 1998 & Phosphate & River waters & Spectrophotometry & $\begin{array}{l}\text { Molybdenum blue method, } \\
\text { comparison to FIA }\end{array}$ & $0-70 \mathrm{mg} \mathrm{L}^{-1}$ & $0.5 \mathrm{mg} \mathrm{L}^{-1}$ & $0.9 \%$ & $18 \mathrm{~h}^{-1}$ \\
\hline 1998 & $\mathrm{Fe}(\mathrm{II})$, phosphate & $\begin{array}{l}\text { Tap waters and ground } \\
\text { waters }\end{array}$ & $\begin{array}{l}\text { Spectrophotometry-diode } \\
\text { array }\end{array}$ & $\begin{array}{l}\text { Sample as carrier, reaction } \\
\text { between Fe(II) and } \\
\text { phenanthroline and reaction } \\
\text { of molybdate for phosphate }\end{array}$ & $\begin{array}{l}0.25-6 \mathrm{mg} \mathrm{L}^{-1} \mathrm{Fe}(\mathrm{II}), \\
0.1-1 \mathrm{mg} \mathrm{L}^{-1} \mathrm{P}\end{array}$ & $\begin{array}{l}0.06 \mathrm{mg} \mathrm{L}^{-1} \mathrm{Fe}(\mathrm{II}) \\
0.02 \mathrm{mg} \mathrm{L}^{-1} \mathrm{P}\end{array}$ & $\begin{array}{l}\text { 1.7\% Fe(II), } \\
4.1 \% \mathrm{P}\end{array}$ & - \\
\hline 1998 & $\mathrm{Fe}(\mathrm{III})$ & Mine waters & Spectrophotometry & $\begin{array}{l}\text { Investigation of the use of } \\
\text { mixing chambers, reaction } \\
\text { between iron and tiron }\end{array}$ & $0-200 \mathrm{mg} \mathrm{L}^{-1}$ & $0.03 \mathrm{mg} \mathrm{L}^{-1}$ & $0.28 \%$ & $24 \mathrm{~h}^{-1}$ \\
\hline 1998 & $\mathrm{Fe}(\mathrm{II})$ & Natural waters & Spectrophotometry & $\begin{array}{l}\text { New strategies of sampling, } \\
\text { reaction between } \mathrm{Fe}(\mathrm{II}) \text { and } \\
\text { phenantroline }\end{array}$ & - & - & $1.1 \%$ & - \\
\hline 1998 & $\begin{array}{l}\mathrm{Co}(\mathrm{II}) \\
\mathrm{Ni}(\mathrm{II})\end{array}$ & $\begin{array}{l}\text { Water samples with known } \\
\text { quantities of the analytes }\end{array}$ & Spectrophotometry & $\begin{array}{l}\text { Kinetic determination based } \\
\text { on different reaction rate } \\
\text { between the analytes and } \\
\text { citrate, colour reaction with } \\
\text { PAR }\end{array}$ & $0-10 \mathrm{mg} \mathrm{L}^{-1}$ & $\begin{array}{l}0.20 \mathrm{mg} \mathrm{L}^{-1} \\
0.14 \mathrm{mg} \mathrm{L}^{-1}\end{array}$ & $1.20 \%$ & $11 \mathrm{~h}^{-1}$ \\
\hline 1997 & Ammonia & $\begin{array}{l}\text { Water samples and } \\
\text { effluent streams }\end{array}$ & Spectrophotometry & $\begin{array}{l}\text { Based on the reaction of } \\
\text { ammonia with hypochlorite } \\
\text { forming monochloramine } \\
\text { that reacts with phenol } \\
\text { reagent (blue } \\
\text { indophenol-type) }\end{array}$ & $0-50 \mathrm{mg} \mathrm{L}^{-1}$ & $0.36 \mathrm{mg} \mathrm{L}^{-1}$ & $1.80 \%$ & $16 h^{-1}$ \\
\hline 1997 & $\begin{array}{l}\text { Ammonium, nitrite, } \\
\text { orthophosphate }\end{array}$ & Waste waters & Spectrophotometry & $\begin{array}{l}\text { Bromothymol blue for } \\
\text { ammonium, Griess reaction } \\
\text { for nitrite, vanadomolybdate } \\
\text { for orthophosphate }\end{array}$ & $\begin{array}{l}2-60\left(\mathrm{NH}_{4}{ }^{+}\right), 0.5-25 \\
\left(\mathrm{NO}_{2}^{-}\right), 0.5-50 \\
\left(\mathrm{PO}_{4}^{3-}\right) \mathrm{mg} \mathrm{L}^{-1}\end{array}$ & - & - & - \\
\hline 1997 & $\begin{array}{l}\text { Phosphate } \\
\text { Silicate }\end{array}$ & Urban waste water & Spectrophotometry & $\begin{array}{l}\text { Reaction with molybdate } \\
\text { eliminating mutual } \\
\text { interference with } \\
\text { appropriated acidity and } \\
\text { segmentation with oxalic } \\
\text { acid }\end{array}$ & $\begin{array}{l}0-12 \mathrm{mg} \mathrm{L}^{-1} \mathrm{P} \\
0-36 \mathrm{mg} \mathrm{L}^{-1} \mathrm{Si}\end{array}$ & $\begin{array}{l}0.2 \mathrm{mg} \mathrm{L}^{-1} \mathrm{P} \\
0.9 \mathrm{mg} \mathrm{L}^{-1} \mathrm{Si}\end{array}$ & $\begin{array}{l}1.38 \% \\
3.87 \%\end{array}$ & $23 /\left(46 h^{-1}\right)$ \\
\hline 1997 & Orthophosphates & $\begin{array}{l}\text { Natural and waste } \\
\text { waters }\end{array}$ & Spectrophotometry & $\begin{array}{l}\text { Study of three methods with: } \\
\text { vanadomolybdate, malachite } \\
\text { green, molybdenum blue }\end{array}$ & $\begin{array}{l}0-18,0-0.4 \\
0-4 \mathrm{mg} \mathrm{P} \mathrm{L}^{-1} \\
\text { (respectively) }\end{array}$ & $0.15,0.01,0.01 \mathrm{mg} \mathrm{P} \mathrm{L}^{-1}$ & $\begin{array}{l}2.1 \%, 18 \% \\
1.7 \%\end{array}$ & $30 \mathrm{~h}^{-1}$ \\
\hline \multirow[t]{2}{*}{1997} & $\mathrm{Fe}(\mathrm{III})$ & Potable water & $\begin{array}{l}\text { Atomic absorption } \\
\text { spectrophotometry }\end{array}$ & $\begin{array}{l}\text { Without the } \\
\text { preconcentration step }\end{array}$ & $1-20 \mathrm{mg} \mathrm{L}^{-1}$ & $0.088 \mathrm{mg} \mathrm{L}^{-1}$ & $2.00 \%$ & $6 \mathrm{~h}^{-1}$ \\
\hline & & & & $\begin{array}{l}\text { Preconcentration in a Chelex } \\
\text { resin }\end{array}$ & $0.02-0.4 \mathrm{mg} \mathrm{L}^{-1}$ & $0.006 \mathrm{mg} \mathrm{L}^{-1}$ & $4.80 \%$ & \\
\hline 1997 & $\mathrm{Fe}(\mathrm{III})$ & Water samples & Spectrophotometry & $\begin{array}{l}\text { Based on thiocyanate } \\
\text { reaction, modelling through } \\
\text { a neural network system } \\
\text { using genetic algorithm }\end{array}$ & $5-20 \mathrm{mg} \mathrm{L}^{-1}$ & - & - & $110 \mathrm{~h}^{-1}$ \\
\hline 1997 & $\begin{array}{l}\mathrm{Cr}(\mathrm{VI}) \\
\operatorname{Cr}(\mathrm{III})\end{array}$ & $\begin{array}{l}\text { Tap water, lake water and sea } \\
\text { water }\end{array}$ & Spectrophotometry & $\begin{array}{l}\text { Reaction product of } \mathrm{Cr}(\mathrm{VI}) \\
\text { with diphenylcarbazide } \\
\text { extracted to a wet film, } \\
\text { oxidation of } \mathrm{Cr}(\mathrm{III}) \\
\text { (determination by difference } \\
\text { with total chromium) }\end{array}$ & $2.0 \mathrm{mg} \mathrm{L}^{-1} \mathrm{Cr}(\mathrm{VI})$ & $2.0 \mathrm{mg} \mathrm{L}^{-1} \mathrm{Cr}(\mathrm{VI})$ & $2.80 \%$ & $17 \mathrm{~h}^{-1}$ \\
\hline 1996 & Ammonium & $\begin{array}{l}\text { Urban waste water } \\
\text { samples, aqueous } \\
\text { extracts of atmospheric } \\
\text { aerosols }\end{array}$ & $\begin{array}{l}\text { Spectrophotometry-diode } \\
\text { array }\end{array}$ & $\begin{array}{l}\text { Formation of ammonia that } \\
\text { diffuses through an } \\
\text { hydrophobic membrane into } \\
\text { an acid-base indicator }\end{array}$ & $0-60 \mathrm{mg} \mathrm{L}^{-1}$ & $2 \mathrm{mg} \mathrm{L}^{-1}$ & $2.50 \%$ & - \\
\hline 1996 & Ammonium & Waste water & Conductimetry & $\begin{array}{l}\text { Coupled of a selection valve } \\
\text { with a injection valve for } \\
\text { sample preconcentration } \\
\text { with a gas diffusion unit }\end{array}$ & $0-180 \mathrm{mg} \mathrm{L}^{-1}$ & $0.1 \mathrm{mg} \mathrm{L}^{-1}$ & $3.0 \%$ & - \\
\hline
\end{tabular}




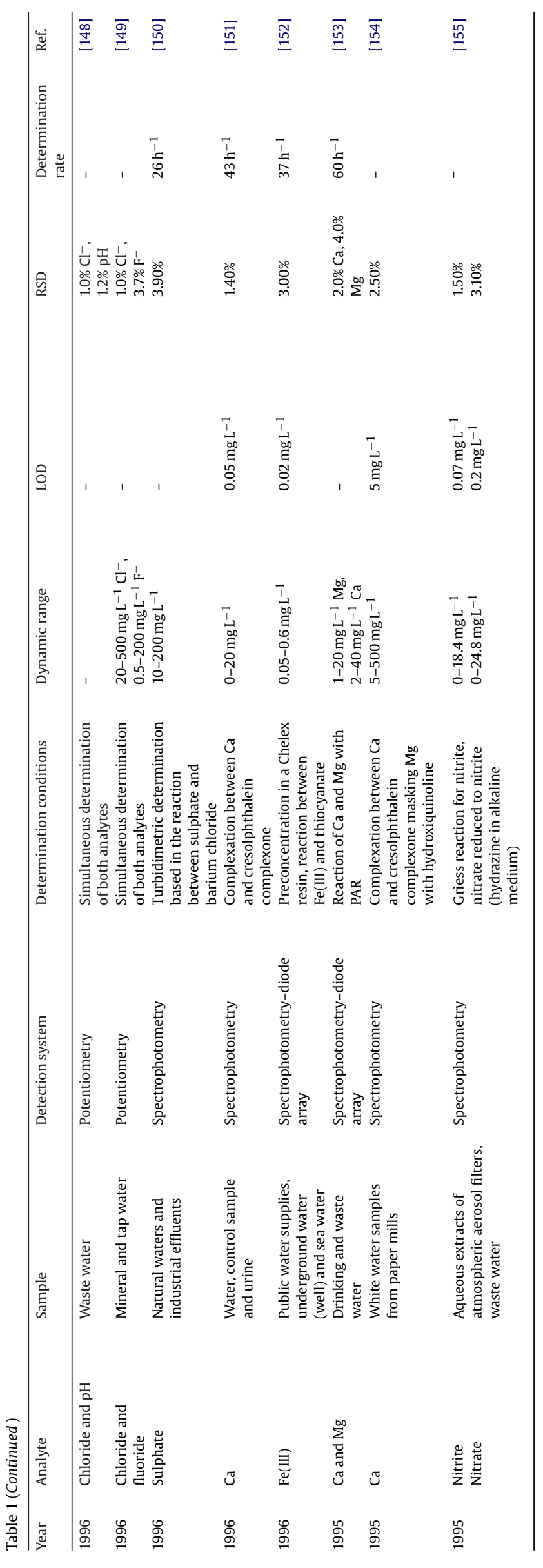

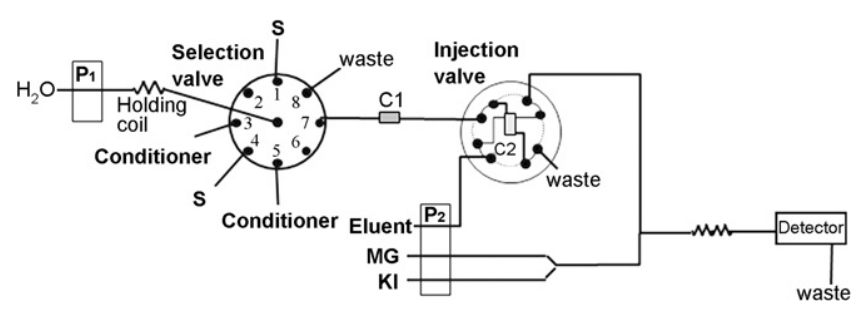

Fig. 3. Schematic representation of a hybrid SIA-FI system for the spectrophotometric determination of lead in water samples with in-line preconcentration of lead and elimination of interferences (adapted from [81]): $\mathrm{Pi}$ - peristaltic pumps, $\mathrm{S}$ - sample, C1 - column filled with an anionic resin for interference elimination, C2 - column filled with a cationic resin for lead preconcentration, MG - malachite green, Eluent - nitric acid solution for eluting the preconcentrated lead.

There were also at least two references to coupling SIA with HPLC $[49,54]$ showing how effective SIA can be in preparing the sample prior to injection. In the work described by Burakham et al. [49], SIA is responsible not only for the preconcentration step but also for the selection of reagents in the different metals determination. In the work described by Sabarudin et al. [58], a solid phase extraction was carried out in a SIA method prior to injection, which enabled the application to waste waters.

In combining SIA with spectrophotometric determinations, different approaches were observed. In some cases, when gaseous analytes were involved, the separation was obtained using a hydrophobic membrane $[69,146]$. In other cases, the separation involved an extraction, either liquid-liquid $[41,54,73]$ or solid-liquid [31,33,81,93,118,152]. These solid-liquid extractions used resins and the analyte was retained, even after the colorimetric reaction [31]. In some of these cases, the analyte was not only extracted from the matrix but also preconcentrated before determination, enabling enhanced limits of detection, e.g. $25 \mu \mathrm{g} \mathrm{Pb} \mathrm{L}^{-1}$ [81] (Fig. 3) and $20 \mu \mathrm{g} \mathrm{Fe} \mathrm{L}^{-1}$ [152].

\section{Changes in oxidation state}

The use of in-line chemical treatments aims mainly to change the oxidation state of a specific analyte. For some determinations it is important to know the oxidation state and/or to convert the analyte to the same oxidation state. This is particularly important in the determination of metals such as chromium [61,72,104,145]. In this case, the in-line treatment aimed for either the oxidation of chromium(III) to chromium(VI) $[38,61,104,145]$, or the reduction of chromium(VI) to chromium(III) [72] depending upon the appropriate oxidation state for the determination. The result was a bi-parametric determination of chromium (III) and chromium (VI).

The same was observed in a couple of iron determinations $[103,127]$, where the reduction of iron(III) to iron(II) resulted in the determination of both ionic forms. Other papers that describe the determination of iron also reported the previous iron reduction but present the results as total amount of iron [59,74].

As for manganese, there was only one description of the determination of both manganese(II) and manganese(VII) [94] resulting from the reduction of manganese(VII) to manganese(II). In another work, manganese(II) determination involved the oxidation of manganese(II) to permanganate prior to the direct determination of this anion [128]. In all of these works, the inclusion of either several reagents or devices in the SI system reinforces the versatility attributed to sequential injection analysis, by performing multideterminations with the same basic configuration.

Oxidation and reduction treatments were not limited to the determination of metals as the determination of nitrite and nitrate is also very common $[96,107,108,116,117,132,155]$. In all these works, nitrate is reduced to nitrite, most of them involving a cadmium column $[96,107,108,116,117,132]$ once again emphasising the easy assembly of a device (column) to a SI system. 


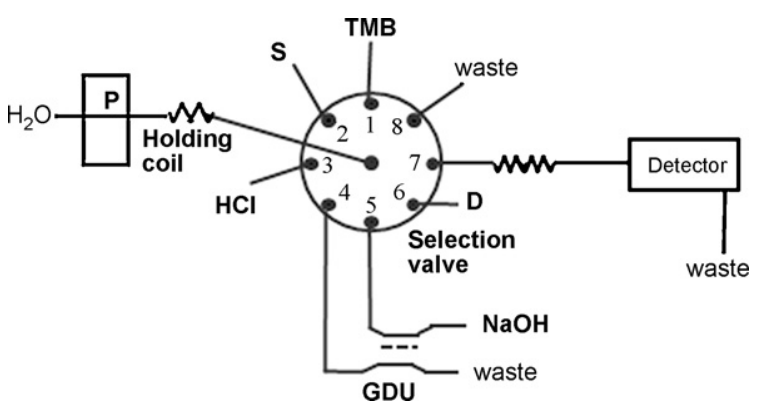

Fig. 4. Schematic representation of a SIA system for the spectrophotometric determination of chlorine with or without matrix separation (adapted from $[44,69]$ ): $P$ - peristaltic pump, S - sample, TMB - tetramethylbenzidine reagent used for the determination without separation, GDU - gas diffusion unit, D - dianisidine reagent used for the determination with gas diffusion separation.

There was one exception to the use of the cadmium column as Oms et al. [155] described the reduction with hydrazine. In the end, most of these works represent a bi-parametric determination of both anions $[96,116,117,132,155]$ but a couple present the results as total nitrogen [107,108]. The work by Thomas et al. [140] described the determination of nitrite and total nitrogen (obtained from sample digestion) but made no reference to the value of nitrate.

Another application of an in-line oxidation process for determination of two oxidation states was observed in the work by van Staden et al. [85]. The authors described the determination of both bromine and bromide; while the value of bromine was obtained by the direct determination, for bromide it was calculated by the difference with total bromine (after oxidation of bromide).

Another application of the oxidation/reduction process is the determination of chlorine described by Mesquita and Rangel [69] in which hypochlorite was reduced to chlorine in order to diffuse through a hydrophobic membrane and subsequently oxidised back to hypochlorite for the colorimetric determination. In Fig. 4, a sequential injection manifold is presented in which chlorine could be determined with either a selective reagent (tetramethylbenzidine) or with matrix separation and a non-selective reagent (dianisidine).

\section{Metals determination}

The range of analytes covered by the works listed in Table 1 is fairly extensive, but a little more than half (56\%), described the determination of metal cations in water. The importance of metal cations in water is mainly related to their toxicity, which depends upon their concentration. Most regulations for water quality have strict limits for metals.

Among these, iron is the most determined metal in waters, if the two possible ionic forms are considered [49,59,71,74,83,103,127, $135-137,143,144,152]$. This fact can be explained as iron determination can be fairly easy to obtain with spectrophotometric detection, due to well-known reactions. It seems pertinent to highlight some of those described works as they constitute a significantly different approach to the determination. That was the case of the work developed by Makchit et al. [71]; the authors designed a homemade sequential injection system providing valuable information for "a do it yourself SI manifold". Other examples are described by Vieira et al. [137] and Mas et al. [135], involving different strategies of sampling and the use of sample as carrier stream.

For the determination of chromium, spectrophotometric $[60,61,91,104,145]$, atomic absorption $[39,72,73,114]$ and ICP detection [50] was used. All the methodologies described with AAS detection used a preconcentration step.

Metals such as copper [48-51,59,74,92,101,102,113], cadmium $[40,47,50,51,83,90,99-101,126]$ and lead $[40,41,50,51,58,81-83$,
101,102,129] were mainly determined with ICP $[50,51,58,102]$ and AAS $[41,47,48,88,99,100,126,129]$ because these metals are found at very low concentrations in waters. In fact, there was only one spectrophotometric method described for cadmium [83], two for lead $[81,82]$ and four for copper $[59,74,92,130]$ as spectrophotometric determinations normally imply higher detection limits.

A rather different approach was the use of voltammetric determination of several metals by Chuanuwatanakul et al. in the determination of lead, cadmium and zinc [40] and by Suteerapataranon et al. in the determination of copper, cadmium and lead [101].

Mercury is a heavy metal with very low concentrations in natural waters, so highly sensitive detection methods are required for its determination. Cold-vapour AAS was exclusively applied for the determination of mercury $[80,112,124,125]$ providing detection limits as low as $10 \mathrm{ng} \mathrm{L}^{-1}$ [124]. A new alternative with a lab-on-valve micro-scale vapour generation chamber coupled with atomic fluorescence detection was recently described by Yu et al. [46], exploiting new applications of the cold-vapour generation. There were only a few descriptions of spectrophotometric detection $[83,93]$ for the determination of mercury and, despite the use of a preconcentration procedure, the detection limit obtained was only about $1 \mathrm{mg} \mathrm{L}^{-1}$.

Other metals such as manganese, nickel and cobalt, although not commonly determined in water samples, may be indicators of contamination. Taljaard and van Staden [138] described the determination of nickel and cobalt based on the same colorimetric reaction but with different kinetics for each analyte, while the individual determination of nickel involving separation of the analyte from the sample with AAS detection has been described $[56,113]$.

Spectrophotometric method have been described for the determination of manganese, either as manganese(II) [128] or manganese(II) and (VII) [94].

Other metals (silver, uranium [51], barium [45], molybdenum [102] and vanadium [51,102]) were determined using multiparametric methodologies involving ICP-AES detection [51], ICP-MS [102] and potenciometric detection [45].

Only one method has been described for the determination of strontium: the aim was to develop a new extraction procedure. In this work, by Miró et al. [105], sequential injection was used to carry out the wet film extraction procedure prior to determination of radioactive strontium by low-background gas-flow proportional counter.

Recently, sequential injection was coupled with an array of potentiometric detectors described as an "electronic tongue" $[34,35,45,59]$. The aim of this technique is to mimic the human sense of taste, and this approach involves the simultaneous determination of metal ions such as sodium, potassium, calcium, magnesium and barium. In order to "taste the sample", a mathematical model had to be created based in the determination of different concentrations of the different ions, a process called training. Sequential injection was used to automate this training step.

\section{Non-metals determination}

\section{Anions}

The determination of anions represents about $46 \%$ of the papers listed in Table 1, which can be easily explained as most macro nutrients present in waters are included in this category.

Spectrophotometry was the most widely used method of detection, although there were also quite a few methods that involved electrochemical detection. Almost 20\% each of the listed references, described the determination of nitrite $[67,96,107,118$, $116,117,132,131,140,155]$ and phosphate $[52,77,107,120,119,135,134$, 140-142]. For monitoring aquatic media, it is important not only the determination of these anions individually, but also the ratio 
between them, currently called N:P content. In fact, a couple of the mentioned methodologies enabled to obtain that ratio [107,140].

Although nitrate is the ionic form of nitrogen that is biologically available, most nitrate determinations involve its prior reduction to nitrite before detection by the Griess method (see Section 4.4.2). So, in the end, those methodologies described the determination of both nitrate and nitrite [96,107,118,116,132,155].

As a major dissolved component of rain water, sulfate is also commonly found in natural waters. All of the methods described for the determination of sulfate used spectrophotometric detection $[86,116,122,150]$ were based on the turbidimetric reaction between sulfate and barium chloride. Only Lapa et al. [122] described an alternative to turbidity measurement, namely the determination of sulfate by direct UV detection.

Also relevant in water analysis is the determination of chloride due to its aesthetic effects in drinking water and due to salinity measurement in natural waters.

Most of the described works for chloride determination involved potentiometric detection [53,70,148,149]. Furthermore, except for the work by Jakmunee et al. [70], the described methods were multiparametric, reinforcing the versatility of sequential injection analysis. There were also a couple of methods that used spectrophotometric detection, one based on the turbidimetric reaction between chloride and silver nitrate [95], and another using the colorimetric reaction of chloride with thiosulfate [109]. Due to the influence of chloride on the taste of water, one of the recent applications of sequential injection analysis to the training of the "electronic tongues" also included the determination of chloride [34].

The determination of silicate and phosphate are often described together $[120,119,141]$ because detection of both involves similar colorimetric reaction.

Some anions are not significantly present in natural waters and hence are rarely measured. It is the case of the spectrophotometric determination of bromate [75]. As for fluoride, the developed methods described for its determination used potentiometric detection $[32,149]$. There were some works for the determination of sulfide [87], bromide [85], iodide [110] and thiocyanate [123] where the main application was not water samples but other matrices such as effluents [85,123], pharmaceuticals [110] and simulated samples [87].

\section{Other analytes}

Sequential injection techniques were not commonly applied to the analysis of gaseous analytes. The most recent application was to the determination of chlorine $[44,69]$, which has become a significant contaminant due to the excessive use of disinfection products in water treatments. In the work by Mesquita and Rangel [69], the ability of gaseous chlorine to cross through a gas diffusion unit was used to remove it from a water sample prior to colorimetric determination. A different approach was used by Mesquita et al. [44], in which chlorine was oxidised to hypochlorite prior to determination, based on the colorimetric reaction with a specific reagent.

The use of gas diffusion has also been described by van Staden and Taljaard [139] for the determination of ammonia.

Recently the determination of a number of human-health related water quality parameters have been described, e.g. determination of pharmaceuticals [54] in water streams. Their presence results from human consumption and excretion and their environmental impact is yet not fully known but is an area of concern. Other emerging analytes in natural and drinking waters include surfactants (nonionic [30], anionic [65] and cationic [64]). These parameters are the consequence of sources of contamination that were not previously considered, in most cases excess of disinfectants and/or detergents used to clean up the water supplies. The contamination may result by the disinfectants and/or detergents themselves or by their by-products. In fact, there are already contaminants such as linear alkylbenzene sulfonates [30] which result from the search of more biodegradable alternatives to highly toxic disinfectants and/or detergents previously used. Another known source of water contamination, namely ground water, is the excess use of pesticides. New pesticides are developed regularly resulting in new contaminants to search in water such as methyl parathion [29], an insecticide.

\section{Off-line pre-treatment}

Some of the previously described systems were applicable to both untreated samples or to samples subject to a previous off-line treatment (for example filtration of waste waters), depending on the sample characteristics. A little over $45 \%$ of all the publications have no reference of any treatment or clearly state that none was made. Some determinations, namely for metals, imply acidification of the sample at the time of collection as part of the sampling procedure. Spiking the samples was made to enable the determination within the described dynamic range and not required for the methodology to be applied, so it was also not classified as previous treatment.

In about $30 \%$ of the publications, the described methodology required a previous treatment. Most of those mentioned previous treatments were as simple as a filtration of the sample when waste water was involved $[64,67,91,118,119,141,142,146]$. In the work by Nyman and Ivaska [154], centrifugation of the sample was used with the same purpose. Other pre-treatments were more complex such as acidic digestion of the sample for the determination of total iron [71] and total mercury [93]. Although extraction procedures were already mentioned in Section 3.4.1 as in-line pre-treatments, in the work by Roerdink and Aldstadt [66] for the determination of $p$-arsenilic acid, solid phase extraction was used as a previous treatment.

\section{Conclusions}

The sequential injection concept proved to be a suitable choice for meeting the purpose of turning water analysis into a faster, more efficient and automatic procedure. The use of sequential injection analysis enabled, with the same basic equipment and configuration, to perform a wide variety of determinations and unit operations. This can be achieved just by using different reagents on the ports of the selection valve or then by coupling devices such as gas diffusion/dialysis systems, packed columns or mixing chambers. These approaches can also be efficient tools for analyte speciation.

As SIA systems are necessarily controlled by computer, they could be further explored towards intelligent automated systems. If a feedback system is implemented and a suitable program is developed, the operation conditions (for example injection volumes, flow-rates) could be readjusted according to the first sample reading. This way, the same configuration could be used for a wide variety of concentration ranges and thus be applicable to different types of waters or then to control a waste water treatment process.

Being composed of relatively small, but robust equipment, it can be used for at-line determinations. This is also possible due to the low reagent consumption and effluent production of SIA systems.

\section{Acknowledgements}

R.B.R. Mesquita thanks to Fundação para a Ciência e a Tecnologia (FCT) the grant SFRH/BPD/41859/2007. The authors also thank to FCT financial support through project PTDC/AMB/64441/2006. 


\section{References}

[1] Water Frame Directive - directive 2000/60/EC of the parliament and of the council, Official Journal of the European Communities L 327/1, of 23 October 2000, establishing a framework for Community action in the field of water policy.

[2] R. Greenwood, G.A. Mills, B. Roig, Trends Anal. Chem. 26 (2007) 263.

[3] EPA - United States Environmental Protection Agency, July 2007 (last access), Current drinking water standards - List of contaminants. http://www.epa.gov/safewater/mcl.html.

[4] S.D. Richardson, Anal. Chem. 79 (2007) 4295.

[5] P.T. Anastas, Crit. Rev. Anal. Chem. 29 (1999) 167.

[6] J. Rủžička, G.D. Marshall, Anal. Chim. Acta 237 (1990) 329.

[7] J. Rủžička, E.H. Hansen, Anal. Chim. Acta 78 (1975) 145.

[8] B.F. Reis, M.F. Giné, E.A.G. Zagatto, J.L.F.C. Lima, R.A. Lapa, Anal. Chim. Acta 293 (1994) 129.

[9] V. Cerdà, J.M. Estela, R. Forteza, A. Cladera, E. Becerra, P. Altimira, P. Sitjar, Talanta 50 (1999) 695.

[10] R.A.S. Lapa, J.L.F.C. Lima, B.F. Reis, J.L.M. Santos, E.A.G. Zagatto, Anal. Chim. Acta 466 (2002) 125.

[11] J. Rủžička, Analyst 125 (2000) 1053.

[12] J. Rǔžička, L. Scampavia, Anal. Chem. 71 (1999) 257A.

[13] V. Cerdà, A. Cerdà, A. Cladera, M.T. Oms, F. Mas, E. Gómez, F. Bauzá, M. Miro, R. Forteza, J.M. Estela, Trends Anal. Chem. 20 (2001) 407.

[14] M. Miró, J.M. Estela, V. Cerda, Talanta 60 (2003) 867.

[15] M. Miró, J.M. Estela, V. Cerda, Talanta 62 (2004) 1.

[16] M. Miró, J.M. Estela, V. Cerda, Talanta 63 (2004) 201

[17] A.F. Dŭnec, M. Cheregi, J.M. Calatayud, J.V.G. Mateo, H.Y.A. Enein, Crit. Rev. Anal. Chem. 31 (2001) 191.

[18] A.F. Dŭnec, M. Cheregi, J.M. Calatayud, J.V.G. Mateo, H.Y.A. Enein, Crit. Rev. Anal. Chem. 33 (2003) 57.

[19] V. Cerda, J.M. Estela, Int. J. Environ. Anal. Chem. 85 (2005) 231.

[20] J. Wang, E.H. Hansen, Trends Anal. Chem. 22 (2003) 836.

[21] Y. Wang, M.-L. Chen, J.-H. Wang, Appl. Spectrosc. Rev. 42 (2007) 103.

[22] S. Armenta, S. Garrigues, M. de la Guardia, Trends Anal. Chem. 26 (2007) 775.

[23] M. Gallignani, M.R. Brunetto, Talanta 64 (2004) 1127.

[24] M. Burguera, J.L. Burguera, Spectrochim. Acta, Part B 62 (2007) 884

[25] A. Gutés, F. Céspedes, M. del Valle, Anal. Chim. Acta 600 (2007) 90.

[26] S. Gray, G. Hanrahan, I. McKelvie, A. Tappin, F. Tse, P. Worsfold, Environ. Chem. $3(2007) 3$.

[27] S. Motomizu, Z.-H. Li, Talanta 66 (2005) 332.

[28] T.P. Rao, P. Metilda, J.M. Gladis, Crit. Rev. Anal. Chem. 35 (2007) 247.

[29] L.B.O. Santos, J.C. Masini, Anal. Chim. Acta 606 (2008) 209.

[30] J.R. Jiménez, M.D.L. Castro, Electrophoresis 29 (2008) 590.

[31] G. Chen, D. Yuan, Y. Huang, M. Zhang, M. Bergman, Anal. Chim. Acta 620 (2008) 82.

[32] A.C.L. Conceição, M.M.C. Santos, M.L.S.S. Gonçalves, Talanta 76 (2008) 107.

[33] J. Ma, D. Yuan, Y. Liang, Mar. Chem. 111 (2008) 151.

[34] A. Ipatov, N. Abramova, A. Bratov, C. Domínguez, Sens. Actuators, B 131 (2008) 48.

[35] D. Calvo, A. Durán, M. Valle, Sens. Actuators, B 131 (2008) 77.

[36] A. Ipatov, N. Abramova, A. Bratov, Talanta 77 (2008) 581.

[37] R.B.R. Mesquita, A.O.S.S. Rangel, J. Braz. Chem. Soc. 19 (2008) 1171.

[38] A.-M. Zou, X.-W. Chen, M.-L. Chen, J.-H. Wang, J. Anal. At. Spectrom. 23 (2008) 412.

[39] A.-M. Zou, X.-Y. Tang, M.-L. Chen, J.-H. Wang, Spectrochim. Acta, Part B 63 (2008) 607.

[40] S. Chuanuwatanakul, W. Dungchai, O. Chailapakul, S. Motomizu, Anal. Sci. 24 (2008) 589.

[41] A.N. Anthemidis, Talanta 77 (2008) 541

[42] R. Zang, H. Nakajima, N. Soh, K. Nakano, T. Masadome, K. Nagata, K. Sakamoto, T. Imato, Anal. Chim. Acta 600 (2007) 105.

[43] L.B.O. Santos, J.C. Masini, Talanta 72 (2007) 1023.

[44] R.B.R. Mesquita, M.L.F.O.B. Noronha, A.I.L. Pereira, A.C.F. Santos, A.F. Torres, V. Cerdà, A.O.S.S. Rangel, Talanta 72 (2007) 1186.

[45] D. Calvo, M. Größl, M. Cortina, M. del Valle, Electroanalysis 19 (2007) 644.

[46] Y.-L. Yu, Z. Du, J.-H. Wang, J. Anal. At. Spectrom. 22 (2007) 650.

[47] A.-M. Zou, M.-L. Chen, Y. Shu, M. Yang, J.-H. Wang, J. Anal. At. Spectrom. 22 (2007) 392.

[48] Y. Yong-Liang, D. Zhou, W. Jian-Hua, Chin. J. Anal. Chem. 35 (2007) 431.

[49] R. Burakham, S. Srijaranai, K. Grudpan, J. Sep. Sci. 30 (2007) 2614.

[50] R.K. Katarina, N. Lenghor, S. Motomizu, Anal. Sci. 23 (2007) 343

[51] A. Sabarudin, N. Lenghor, M. Oshima, L. Hakim, T. Takayanagi, Y.-H. Gao, S. Motomizu, Talanta 72 (2007) 1609.

[52] C. Frank, F. Schroeder, R. Ebinghaus, W. Ruck, Microchim. Acta 154 (2006) 31.

[53] M. Cortina, A. Duran, S. Alegret, M. del Valle, Anal. Bioanal. Chem. 385 (2006) 1186.

[54] J.B. Quintana, M. Miró, J.M. Estela, V. Cerdà, Anal. Chem. 78 (2006) 2832.

[55] A.D. Idowu, P.K. Dasgupta, Z. Genfa, K. Toda, Anal. Chem. 78 (2007) 7088.

[56] X. Long, M. Miró, R. Jensen, E.H. Hansen, Anal. Bioanal. Chem. 386 (2006) 739.

[57] A.N. Anthemidis, Spectrosc. Lett. 39 (2007) 699.

[58] A. Sabarudin, N. Lenghor, Y. Living, Y. Furusho, S. Motomizu, Spectrosc. Lett. 39 (2006) 669.

[59] S. Ohno, N. Teshima, T. Sakai, K. Grudpan, M. Polasek, Talanta 68 (2006) 527.

[60] V. Gómez, M.S. Larrechi, M.P. Callao, Anal. Chim. Acta 571 (2006) 129.

[61] J. Wang, B. Xue, Anal. Sci. 22 (2006) 1233.
[62] G. Liu, S.L. Riechers, C. Timchalk, Y. Lin, Electrochem. Commun. 7 (2005) 1463.

[63] S. Feng, X. Chen, J. Fan, Z. Hu, Int. J. Environ. Anal. Chem. 85 (2005) 63.

[64] M.L.C. Passos, M.L.M.F.S. Saraiva, J.L.F.C. Lima, Anal. Sci. 21 (2005) 1509.

[65] R. Burakham, S. Lapanantnoppakhun, J. Jakmunee, K. Grudpan, Talanta 68 (2005) 416.

[66] A. Roerdink, J.H. Aldstadt III, Anal. Chim. Acta 539 (2005) 181

[67] M. Baeza, J. Bartrolí, J. Alonso, Talanta 68 (2005) 245.

[68] L.B.O. Santos, M.S.P. Silva, J.C. Masini, Anal. Chim. Acta 528 (2005) 21.

[69] R.B.R. Mesquita, A.O.S.S. Rangel, Talanta 68 (2005) 268.

[70] J. Jakmunee, L. Patimapornlert, S. Suteerapataranon, N. Lenghor, K. Grudpan, Talanta 65 (2005) 789.

[71] J. Makchit, S. Kruanetr, P. Prasertgitwatana, T. Lelasattarathkul, S. Liawruangrath, S. Upalee, W. Oungpipat, Instrum. Sci. Technol. 33 (2005) 565.

[72] X. Long, M. Miró, E.H. Hansen, J. Anal. At. Spectrom. 20 (2005) 1203.

[73] X. Long, M. Miró, E.H. Hansen, Anal. Chem. 77 (2005) 6032.

[74] I.P.A. Morais, M.R.S. Souto, A.O.S.S. Rangel, J. AOAC Int. 88 (2005) 639

[75] J.F. van Staden, L.V. Mulaudzi, R.I. Stefan, Talanta 64 (2004) 1196.

[76] A. Economou, D.G. Themelis, H. Bikou, P.D. Tzanavaras, P.G. Rigas, Anal. Chim. Acta 510 (2004) 219.

[77] F. Mas-Torres, J.M. Estela, M. Miró, A. Cladera, V. Cerdà, Anal. Chim. Acta 510 (2004) 61.

[78] R.B.R. Mesquita, A.O.S.S. Rangel, Anal. Sci. 20 (2004) 1205.

[79] C. Brach-Papa, B. Coulomb, C. Branger, A. Margaillan, F. Theraulaz, P. Loot, J.-L. Boudenne, Anal. Bioanal. Chem. 378 (2004) 1652.

[80] A.N. Anthemidis, G.A. Zachariadis, J.A. Stratis, Talanta 64 (2004) 1053

[81] R.B.R. Mesquita, S.M.V. Fernandes, A.O.S.S. Rangel, Talanta 62 (2004) 395.

[82] N.Z. Aracama, A.N. Araújo, R. Perez-Olmos, Anal. Sci. 20 (2004) 679.

[83] J.F. van Staden, R.E. Taljaard, Talanta 64 (2004) 1203.

[84] P.J. Fletcher, J.F. van Staden, Anal. Chim. Acta 485 (2003) 187.

[85] J.F. van Staden, L.V. Mulaudzi, R.I. Stefan, Anal. Bioanal. Chem. 375 (2003) 1074

[86] I.P.A. Morais, M.R.S. Souto, T.I.M.S. Lopes, A.O.S.S. Rangel, Water Res. 37 (2003) 4243.

[87] M.S.P. Silva, C.X. Galhardo, J.C. Masini, Talanta 60 (2003) 45.

[88] S.M.Z. Al-Kindy, F.E.O. Suliman, S.B. Salama, M. Aoudia, S.N. Al-Bahry, H.S. AlBahlany, Anal. Sci. 19 (2003) 737.

[89] S.M.Z. Al-Kindy, F.O. Suliman, S.B. Salama, Microchem. J. 74 (2003) 173

[90] M. Miró, S. Jończyk, J. Wang, E.H. Hansen, J. Anal. At. Spectrom. 18 (2003) 89.

[91] M.A.S. Pressman, J.H. Aldstadt, Microchem. J. 74 (2003) 47.

[92] P. Rumori, V. Cerdà, Anal. Chim. Acta 486 (2003) 227.

[93] R.P. Sartini, E.C. Vidotti, C.C. Oliveira, Anal. Sci. 19 (2003) 1653.

[94] J.F. van Staden, L.V. Mulaudzi, R.I. Stefan, Anal. Chim. Acta 499 (2003) 129

[95] R.B.R. Mesquita, S.M.V. Fernandes, A.O.S.S. Rangel, J. Environ. Monit. 4 (2002) 458.

[96] Z. Legnerová, P. Solich, H. Sklenářová, D. Šatínský, R. Karlíček, Water Res. 36 (2002) 2777.

[97] G. Armas, M. Miró, J.M. Estela, V. Cerdà, Anal. Chim. Acta 471 (2002) 173.

[98] C. Brach-Papa, B. Coulomb, J.-L. Boudenne, V. Cerdà, F. Theraulaz, Anal. Chim. Acta 457 (2002) 311.

[99] J. Wang, E.H. Hansen, Anal. Chim. Acta 456 (2002) 283.

[100] J. Wang, E.H. Hansen, J. Anal. At. Spectrom. 17 (2002) 248.

[101] S. Suteerapataranon, J. Jakmunee, Y. Vaneesorn, K. Grudpan, Talanta 58 (2002) 1235.

[102] M.S. Jiménez, R. Velarte, J.R. Castillo, Spectrochim. Acta, Part B 57 (2002) 391

[103] L.V. Mulaudzi, J.F. van Staden, R.I. Stefan, Anal. Chim. Acta 467 (2002) 35.

[104] L.V. Mulaudzi, J.F. van Staden, R.I. Stefan, Anal. Chim. Acta 467 (2002) 51.

[105] M. Miró, E. Gómez, J.M. Estela, M. Casas, V. Cerdà, Anal. Chem. 74 (2002) 826.

[106] G. de Armas, E. Becerra, A. Cladera, J.M. Estela, V. Cerdà, Anal. Chim. Acta 427 (2001) 83.

[107] C.-H. Wu, J. Rủžička, Analyst 126 (2001) 1947.

[108] E.B. Naidoo, J.F. van Staden, Water SA 27 (2001) 355

[109] J.F. van Staden, S.I. Tlowana, Fresenius J. Anal. Chem. 371 (2001) 369.

[110] J.A. Erustes, R. Forteza, V. Cerdà, J. AOAC Int. 84 (2001) 337.

[111] J.A. Erustes, A. Andrade-Eiroa, A. Cladera, R. Forteza, V. Cerdà, Analyst 126 (2001) 451.

[112] O. Elsholz, C. Frank, B. Stachel, H. Reincke, R. Ebinghaus, Anal. Chim. Acta 438 (2001) 251.

[113] J. Wang, E.H. Hansen, Anal. Chim. Acta 435 (2001) 331

[114] M.J. Marqués, A. Morales-Rubio, A. Salvador, M. de la Guardia, Talanta 53 (2001) 1229.

[115] J.F. van Staden, T.A. Merwe, Analyst 125 (2000) 2094

[116] R.A.S. Lapa, J.L.F.C. Lima, I.V.O.S. Pinto, Analusis 28 (2000) 295.

[117] R.A.S. Lapa, J.L.F.C. Lima, I.V.O.S. Pinto, Anal. Sci. 16 (2000) 1157.

[118] M. Miró, A. Cladera, J.M. Estela, V. Cerdà, Analyst 125 (2000) 943

[119] C.X. Galhardo, J.C. Masini, Anal. Chim. Acta 417 (2000) 191.

[120] F. Mas-Torres, A. Muñoz, J.M. Estela, V. Cerdà, Int. J. Environ. Anal. Chem. 77 (2000) 185

[121] H. du Plessis, J.F. van Staden, Talanta 52 (2000) 83.

[122] R.A.S. Lapa, J.L.F.C. Lima, I.V.O.S. Pinto, J. Braz. Chem. Soc. 11 (2000) 170

[123] J.F. van Staden, A. Both, Anal. Chim. Acta 403 (2000) 279.

[124] O. Elsholz, C. Frank, B. Matyschok, F. Steiner, O. Wurl, B. Stachel, H. Reincke, M. Schulze R. Ebinghaus, M. Hempel, Fresenius J. Anal. Chem. 366 (2000) 196.

[125] W.E. Doering, R.R. James, R.T. Echols, Fresenius J. Anal. Chem. 368 (2000) 475

[126] Z.-R. Xu, H.-Y. Pan, S.-K. Xu, Z.-L. Fang, Spectrochim. Acta, Part B 55 (2000) 213.

[127] E.B. Naidoo, J.F. van Staden, S. Afr. J. Chem. 53 (2000) 191.

[128] E.B. Naidoo, J.F. van Staden, Fresenius J. Anal. Chem. 370 (2000) 776.

[129] A.N. Araújo, R.C.C. Costa, J.L.F.C. Lima, Anal. Sci. 15 (1999) 991. 
[130] J.F. van Staden, A. Botha, Talanta 49 (1999) 1099.

[131] J.F. van Staden, T.A. Merwe, Microchim. Acta 129 (1998) 33.

[132] A. Cerdà, M.T. Oms, R. Forteza, V. Cerdà, Anal. Chim. Acta 371 (1998) 63.

[133] L.X. Tang, F.J. Rowell, Anal. Lett. 31 (1998) 891.

[134] J.F. van Staden, R.E. Taljaard, Microchim. Acta 128 (1998) 223.

[135] F. Mas, A. Cladera, J.M. Estela, V. Cerdà, Analyst 123 (1998) 1541

[136] T. McCormack, J.F. van Staden, Anal. Chim. Acta 367 (1998) 111.

[137] J.A. Vieira, I.M. Raimundo, B.F. Reis, E.A.G. Zagatto, J.L.F.C. Lima, Anal. Chim Acta 366 (1998) 257.

[138] R.E. Taljaard, J.F. van Staden, Anal. Chim. Acta 366 (1998) 177.

[139] J.F. van Staden, R.E. Taljaard, Anal. Chim. Acta 344 (1997) 281

[140] O. Thomas, F. Théraulaz, V. Cerdà, D. Constant, P. Quevaullier, Trends Anal. Chem. 16 (1997) 419.

[141] F. Mas-Torres, A. Muñoz, J.M. Estela, V. Cerda, Analyst 122 (1997) 1033.

[142] A. Munõz, F. Mas-Torres, A. Munõz, J.M. Estela, V. Cerdà, Anal. Chim. Acta 350 (1997) 21.

[143] E. Rubí, M.S. Jiménez, F.B. Mirabó, R. Forteza, V. Cerdà, Talanta 44 (1997) 553.
[144] J. Garcia, M.L.M.F.S. Saraiva, A.N. Araújo, J.L.F.C. Lima, M. del Valle, M. Poch, Anal. Chim. Acta 348 (1997) 143.

[145] Y. Luo, S. Nakano, D.A. Holman, J. Rủžička, G.D. Christian, Talanta 44 (1997) 1563.

[146] M.T. Oms, A. Cerdà, A. Cladera, V. Cerdà, R. Forteza, Anal. Chim. Acta 318 (1996) 251.

[147] M.T. Oms, A. Cerdà, V. Cerdà, Electroanalysis 8 (1996) 387.

[148] J. Alpízar, A. Crespí, A. Cladera, R. Forteza, V. Cerdà, Lab. Rob. Autom. 8 (1996) 165.

[149] J. Alpízar, A. Crespí, A. Cladera, R. Forteza, V. Cerdà, Electroanalysis 11 (1996) 1051.

[150] J.F. van Staden, R.E. Taljaard, Anal. Chim. Acta 331 (1996) 271

[151] J.F. van Staden, R.E. Taljaard, Anal. Chim. Acta 323 (1996) 75.

[152] E. Rubí, R. Forteza, V. Cerdà, Lab. Rob. Autom. 8 (1996) 149.

[153] E. Gómez, C. Tomás, A. Cladera, J.M. Estela, V. Cerdà, Analyst 120 (1995) 1181

[154] J. Nyman, A. Ivaska, Anal. Chim. Acta 308 (1995) 286.

[155] M.T. Oms, A. Cerdà, V. Cerdà, Anal. Chim. Acta 315 (1995) 321. 